\title{
Investigating the Factors That Contribute to African Easterly Wave Intensity Forecast Uncertainty in the ECMWF Ensemble Prediction System
}

\author{
TRAVIS J. ELLESS ${ }^{\mathrm{a}}$ AND RYAN D. TORN \\ Department of Atmospheric and Environmental Science, University at Albany, State University of New York, Albany, New York
}

(Manuscript received 28 February 2018, in final form 5 February 2019)

\begin{abstract}
Although there have been numerous studies documenting the processes/environments that lead to the intensification of African easterly waves (AEWs), only a few of these studies investigated the effect of those processes or the environment on the predictability of AEWs. Here, the large-scale modulation of AEW intensity predictability is evaluated using the 51-member ECMWF ensemble prediction system (EPS) during an active AEW period (July-September 2011-13). Forecasts are stratified based on the 72-h AEW intensity standard deviation (SD) to evaluate hypotheses for how different processes contribute to large forecast SD. While large and small SD forecasts are associated with similar baroclinic and barotropic energy conversions, forecasts with large SD are characterized by higher relative humidity values downstream of the AEW trough. These areas of higher humidity are also associated with higher precipitation and precipitation SD, suggesting that uncertainty associated with diabatic processes could be linked with large AEW intensity SD. Although water vapor is a strong function of longitude and phase of convectively coupled equatorial waves, the cases with large and small SD are characterized by similar longitude and wave phase, suggesting that AEWs occurring in certain locations or convectively coupled equatorial wave phases are not more or less predictable.
\end{abstract}

\section{Introduction}

African easterly waves (AEWs) are synoptic-scale features that traverse over sub-Saharan Africa and are often associated with significant rainfall via mesoscale convective systems (e.g., Payne and McGarry 1977). Previous observational (e.g., Norquist et al. 1977) and idealized modeling (e.g., Thorncroft and Hoskins 1994) studies indicate that AEWs primarily grow through both barotropic and baroclinic energy conversions, as well as through convective/diabatic processes (e.g., Berry and Thorncroft 2005; Hsieh and Cook 2007; Berry and Thorncroft 2012). These diabatic processes can lead to the intensification of AEWs via the generation of available potential energy, which in turn, increases the baroclinic energy conversion (e.g., Hsieh and Cook 2007) and/or promote the upscale growth of diabatically generated potential vorticity (PV; e.g., Berry and Thorncroft 2005). In a case study, Berry and Thorncroft (2012) suggested that variations in AEW convective

\footnotetext{
${ }^{\text {a }}$ Current affiliation: I. M. Systems Group, Inc., College Park, Maryland.
}

Corresponding author: Travis J. Elless, travis.j.elless@noaa.gov activity resulted in different forecast AEW intensities; however, there are few other studies of how diabatic processes create AEW forecast uncertainty in the literature.

Convection exhibits significant spatial and temporal variability over Africa, which in turn can impact AEWs. Janiga and Thorncroft (2013) determined that the heaviest precipitation rates are found over West Africa and are associated with a deep-convective diabatic heating profile. By contrast, the diabatic heating profile over eastern Africa has a more stratiform profile, while convection is climatologically suppressed over central Africa. The East Africa stratiform heating profile maximizes near $500 \mathrm{hPa}$, allowing for the generation of diabaticaly generated PV to occur at the level of the AEW trough $(700 \mathrm{hPa})$ and potentially accelerate the AEW growth. On the other hand, the deep convective heating profile over West Africa maximizes lower than the East Africa profile and lacks the stratiform cooling below $800 \mathrm{hPa}$, which helps to create diabatic PV in the low levels, strengthening the low-level circulation, but acts to decrease midlevel PV, weakening the 700-hPa trough. As a consequence, regional differences in AEW structure/ intensity are likely to arise due to these different heating profiles generating PV at different levels/rates, and in turn modulate AEW predictability. In addition, convection 
over the African continent is a function of 10-25- and 2560-day intraseasonal features (Janicot and Sultan 2001; Sultan et al. 2003). Variability on these time scales is often linked to convectively coupled equatorial waves such as the Madden-Julian oscillation (MJO; e.g., Zhang 2005) and the convectively coupled Kelvin wave (CCKW; e.g., Straub and Kiladis 2003) respectively. Increased baroclinic growth can occur with enhanced convection during the convectively active phase of these equatorial waves, while their dynamical structure also enhances barotropic growth (Ventrice et al. 2011; Ventrice and Thorncroft 2013), suggesting that the phase of an equatorial wave can enhance AEW intensification through many different pathways.

AEWs are not the only phenomena where convection could impact the predictability of a larger-scale feature. Lorenz (1969) was the first to note the upscale growth of errors during the first hours of model integration. Zhang et al. (2003) demonstrated how differences in smallscale moist processes can lead to variability in baroclinic cyclone position, intensity, and subsequently, precipitation distribution/snowfall at 30-h lead time. More recent studies suggest that the upscale growth of errors from the smallest scales may not be the primary source for these forecast differences. In particular, it appears that convective-scale differences form due to uncertainty in large-scale features which subsequently project onto the smaller scales (e.g., Durran and Gingrich 2014; Durran and Weyn 2016). As a consequence, they suggest that predictability is a function of larger-scale environment.

Other studies of tropical cyclone (TC) predictability further support the notion that environmental differences lead to convective variability. Rios-Berrios et al. (2016a,b) demonstrated how water vapor uncertainty near the TC leads to changes in convection for TCs Katia and Ophelia (2011). Ensemble members with enhanced moisture/convection generated more potential vorticity, which helped to intensify the TC through vortex stretching. Furthermore, Komaromi and Majumdar (2015) noted that European Centre for Medium-Range Weather Forecasts (ECMWF) ensemble prediction system (EPS) forecasts of TCs forming from AEWs were sensitive to the near-storm convective environment. For the majority of their cases, the forecasts exhibited a strong correlation between the 0-h 700-hPa relative humidity (RH) uncertainty and the disturbance (i.e., AEW) forecast intensity variability, such that systems with higher variability in environmental moisture uncertainty were associated with higher circulation variability. Their results suggest that differences in large-scale environmental features played a role in determining forecast predictability.

Early work in AEW predictability highlighted largescale environmental differences could be important to
AEW intensity predictability. Torn (2010) showed that forecasts of a strong AEW were sensitive to downstream thermodynamic variables, such that increasing the $0-\mathrm{h}$ equivalent potential temperature $\left(\theta_{e}\right)$ downstream of the AEW lead to a more intense AEW later in the forecast. While this study suggests that uncertainty in the thermodynamic profile is important to AEW predictability, a larger sample of cases is necessary to understand how the large-scale environment and various processes enhance or limit to AEW predictability.

This study extends the work of Komaromi and Majumdar (2015) and Torn (2010) by investigating the role of large-scale environmental conditions on AEW predictability, measured through forecast intensity standard deviation (SD). Specifically, this study evaluates hypotheses that AEW intensity predictability is a function of the large-scale environmental water vapor (which can be a function of convectively coupled equatorial wave phase), AEW intensity, AEW location, barotropic instability, and/or baroclinic instability using a large dataset of ECMWF ensemble forecasts from 3 years of active AEW activity. One of the unique aspects of this study is the large number of cases analyzed, which goes beyond previous research which looked at only a small number of cases. The data and methods used for our analysis are described in section 2. Section 3 provides summary SD statistics, while differences between high and low SD forecasts are presented in section 4 . Section 5 discusses the impacts of regional convective differences and convectively coupled equatorial waves have on AEW intensity SD. Finally, a summary of the conclusions are presented in section 6.

\section{Methodology}

This study utilizes the AEW forecast climatology described in Elless and Torn (2018) to analyze AEW intensity predictability. A summary of this dataset and methods is provided here; the interested reader is directed to Elless and Torn (2018) for additional details. AEWs that are initially over Africa $\left(5^{\circ}-20^{\circ} \mathrm{N}, 17^{\circ} \mathrm{W}-40^{\circ} \mathrm{E}\right)$ are identified and tracked within the 51-member ECWMF EPS forecasts initialized at 0000 UTC during July-AugustSeptember (JAS) 2011-13 utilizing forecast data interpolated to a $1^{\circ} \times 1^{\circ}$ grid provided by the THORPEX Interactive Grand Global Ensemble (TIGGE) database (Bougeault et al. 2010). This period was chosen because it was characterized by above-average AEW activity [cf. Fig. 1 of Elless and Torn (2018)], which provides ample cases. The algorithm objectively tracks AEWs based on area-averaged $700-\mathrm{hPa}$ curvature vorticity through $120 \mathrm{~h}$. Here, AEW intensity is 
defined as the 600-km-area-averaged 700-hPa curvature vorticity at the objectively identified AEW location.

The predictability of ensemble forecasts can be quantified via the ensemble SD, whereby larger SD corresponds with a less predictable forecast while forecasts with low spread are more predictable (e.g., McMurdie and Ancell 2014).

\section{AEW intensity SD}

ECMWF AEW intensity forecasts exhibit a wide variety of forecast SDs during the 2011-13 forecast period (Fig. 1). At $0 \mathrm{~h}$, intensity forecasts are characterized by a mean SD of $0.17 \times 10^{-5} \mathrm{~s}^{-1}$ with values ranging between 0.07 and $0.34 \times 10^{-5} \mathrm{~s}^{-1}$ Throughout the forecast, the difference between the upper and lower quartile SD cases becomes larger with time. The cases with lowest SD are associated with a fairly constant SD $\left(0.1-0.2 \times 10^{-5} \mathrm{~s}^{-1}\right)$. By contrast, the largest SD cases experience a consistent SD growth [0.1-0.2 $\times$ $10^{-5} \mathrm{~s}^{-1}(24 \mathrm{~h})^{-1}$ ] through $120 \mathrm{~h}$. Given the large variety in forecast $\mathrm{SD}$ values, it is important to investigate if certain systematic large-scale conditions lead to more or less predictable AEW intensity forecasts. One hypothesis is that the large-scale thermodynamic environment and associated AEW convection is associated with AEW intensity predictability (i.e., Torn 2010), such that a more convectively favorable/active environment is associated with an increase in AEW intensity SD. However, largescale baroclinic (aided by moist convection) and barotropic instabilities associated with the African easterly jet (AEJ) can also intensify AEWs (e.g., Norquist et al. 1977; Thorncroft and Hoskins 1994); therefore, it is also important to test hypotheses that these instabilities are also associated with AEW intensity predictability, such that making the AEJ more baroclinically and/or barotropically unstable also favor an increase in AEW intensity SD.

\section{Diagnosis of AEW intensity predictability}

\section{a. High intensity $S D$ versus low intensity $S D$}

The above hypotheses are tested by creating two forecast subsets based on the 72-h intensity SD for the 2011-13 forecast period. One subset consists of the top quintile of SD cases (denoted "high SD") and the other consists of the bottom quintile of SD cases (denoted "low SD") ${ }^{1}$. Composites are then created from the $1^{\circ} \times 1^{\circ} \mathrm{ECMWF}$ forecast fields for each subset by averaging the ensemble-mean

\footnotetext{
${ }^{1}$ In total $20 \%$ of cases equates to a subset size of 32 cases during the 2011-13 forecast period.
}

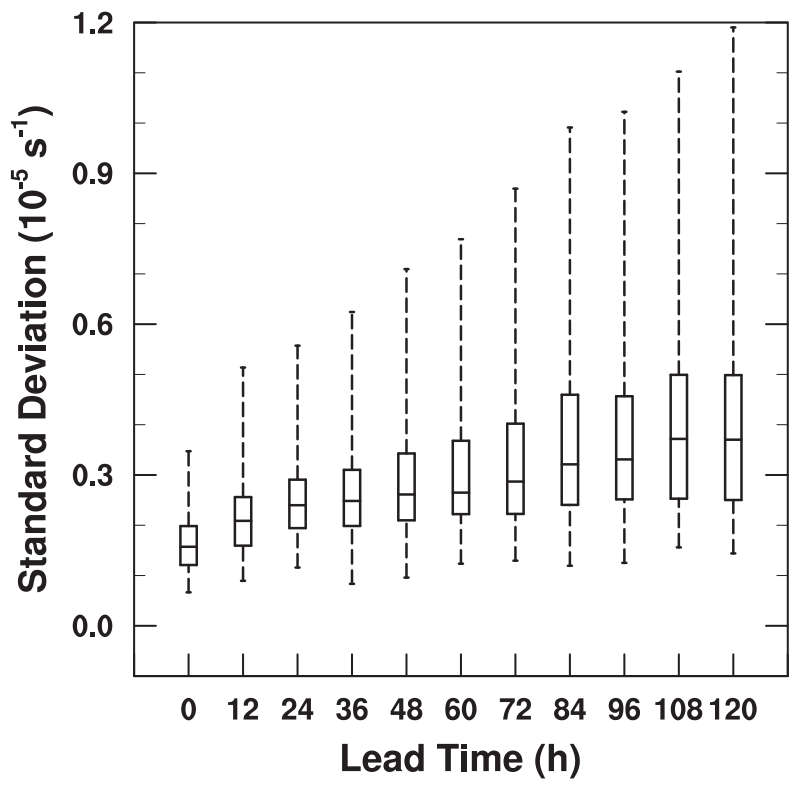

FIG. 1. Box-and-whisker plots of intensity forecast SD for the given lead times during the 2011-13 forecast period, where the box denotes the interquartile of intensity SD and the whiskers the maximum/minimum intensity SD.

fields for each forecast within the subset. The role of AEW properties and the large-scale environment on AEW intensity predictability is evaluated by comparing the two composites. The statistical significance of the composite difference is determined using a bootstrap resampling without replacement approach similar to what is used in Torn et al. (2015) and Rios-Berrios et al. (2016a,b). In this approach, two subsets equal in size to the high and low SD subsets are drawn from the set of all 72 -h forecasts, and the difference between these two random subsets is computed. This process is repeated for 10000 iterations to create a sample distribution where the $95 \%$ confidence interval is determined. The advantage of using this approach is that it retains spatial correlations in the statistical significance calculation. It is also worth noting that the results are similar when the forecasts are subset based on the SD at other lead times (not shown).

Previous studies suggest that the predictability of AEW and TC genesis intensity is linked to the downstream convective environment (e.g., Torn 2010; Komaromi and Majumdar 2015). As a consequence, the hypothesis that the AEW convective environment modulates AEW intensity SD differences is first evaluated by comparing the area-averaged 700-hPa RH and 200-hPa divergence ${ }^{2}$

\footnotetext{
${ }^{2}$ These fields have been used as proxies for the convective environment in previous studies (e.g., Komaromi and Majumdar 2014, 2015; Elless and Torn 2018).
} 

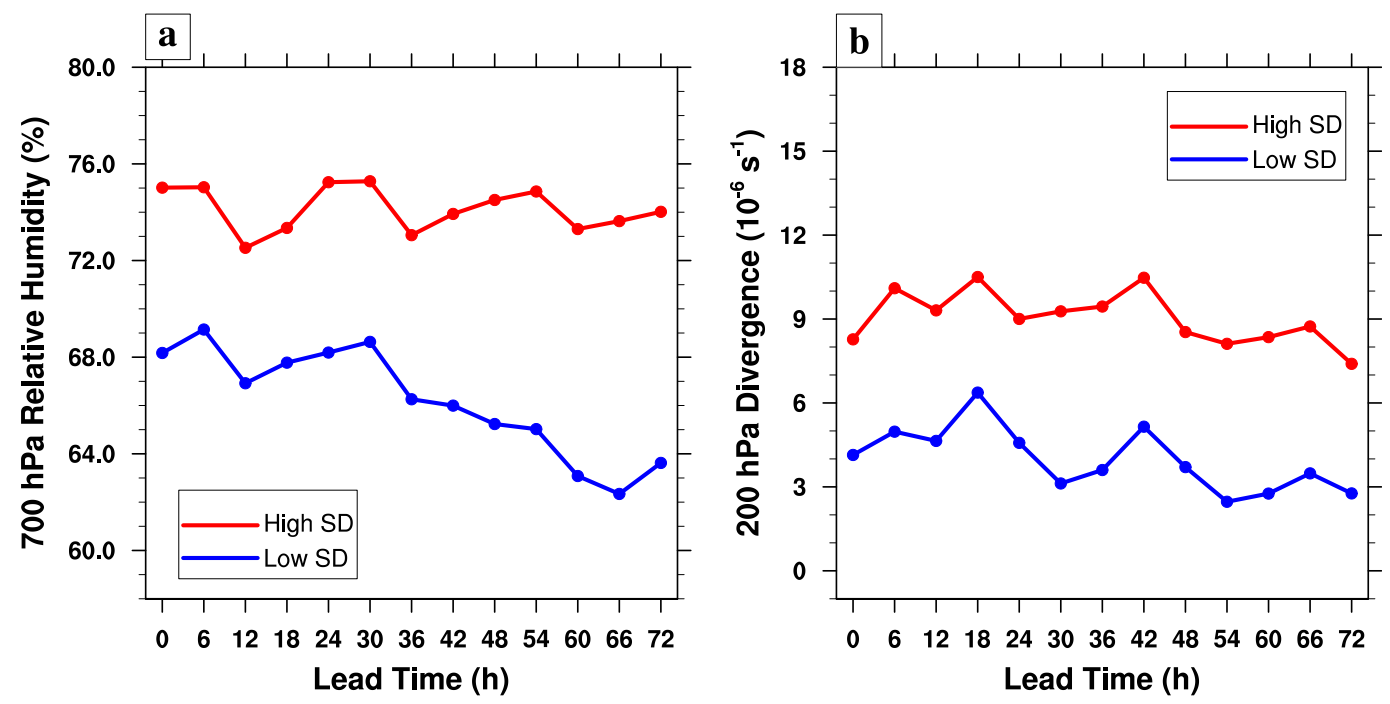

FIG. 2. Composite (a) 700-hPa relative humidity and (b) 200-hPa divergence averaged within $500 \mathrm{~km}$ of the AEW trough center for cases in the high (red) and low (blue) SD subsets during the 2011-13 forecast period. Dots indicated times when the two subsets are statistically different at the $95 \%$ confidence interval using bootstrap resampling.

between the high and low SD cases. The 700-hPa RH provides a measure of midlevel moisture, whereby higher values would help support long-lived convective activity, and hence PV generation, while the 200-hPa divergence is used as a proxy for the integrated deep convection associated with AEW.

The high and low subsets exhibit statistically significant differences in the convective environment during the 2011-13 forecast period. At $0 \mathrm{~h}$, the high SD subsets have a mean $700-\mathrm{hPa}$ RH of $76 \%$, which remains relatively constant through $72 \mathrm{~h}$ (Fig. 2a). A diurnal pattern is also observed in the high SD RH pattern that is consistent with the diurnal pattern of AEW mesoscale convective system (MCS) activity (e.g., Payne and McGarry 1977; Reed et al. 1977; Jackson et al. 2009) with a maximum occurring at lead times that verify at 0000 UTC (i.e., 24, 48, $72 \mathrm{~h}$ ) and a minimum at lead times that verify at 1200 UTC (i.e., $12,36,60 \mathrm{~h}$ ). This pattern suggests that the $\mathrm{RH}$ reflects the diurnal cycle of convection. By contrast, the low SD forecasts are characterized by lower RH during the first $72 \mathrm{~h}$ (Fig. 2a). The low SD forecasts are significantly drier, $68 \%$ at $0 \mathrm{~h}$, with the $\mathrm{RH}$ subsequently decreasing to $63 \%$ by $72 \mathrm{~h}$. These results indicate that the high SD forecasts are located within a more moist environment than the low SD forecasts, which could make it easier to initiate and sustain convection, and could subsequently be responsible for creating larger AEW intensity SD.

Differences in the upper-level divergence can provide further insight into the role of convection differences. The high SD subset has higher 0-h divergence compared to the low SD cases $\left(9 \times 10^{-6} \mathrm{~s}^{-1}\right.$ vs $5 \times 10^{-6} \mathrm{~s}^{-1}$; Fig. 2b), suggesting that the high SD subset has deeper or more extensive convection. Throughout the forecast, the difference between the high and low SD subsets remain fairly constant, suggesting the larger AEW intensity SD cases are more convectively active compared to the low SD cases at all lead times.

While the differences in the near-wave environment support the notion that differences in convection modulate AEW intensity predictability, the location of these differences also appear to be important. Convection is usually observed ahead of the trough center, which would lead to diabatic PV generation downstream of the trough, and hence relocation of the AEW trough downstream (e.g., Berry and Thorncroft 2005; Tomassini et al. 2017). By contrast, the ECMWF ensemble tend to produce convection too close to the trough center (e.g., Elless and Torn 2018), which in turn would generate PV within the AEW and hence intensify it. As a consequence, it is important to also compare the spatial distribution of precipitation within the high and low SD subsets to evaluate whether the high SD forecasts have a different precipitation distribution than the low SD cases.

Spatial differences in precipitation between the high and low SD subsets are examined in a wave-centric framework. For each subset, ensemble forecasts are shifted to an AEW-centric grid (center located at $9^{\circ} \mathrm{N}$, $0^{\circ}$ ) and the composite ensemble-mean precipitation rates are averaged for each case in the subset. During the 2011-13 period, the high and low SD cases are characterized by large ensemble-mean 12-h precipitation 

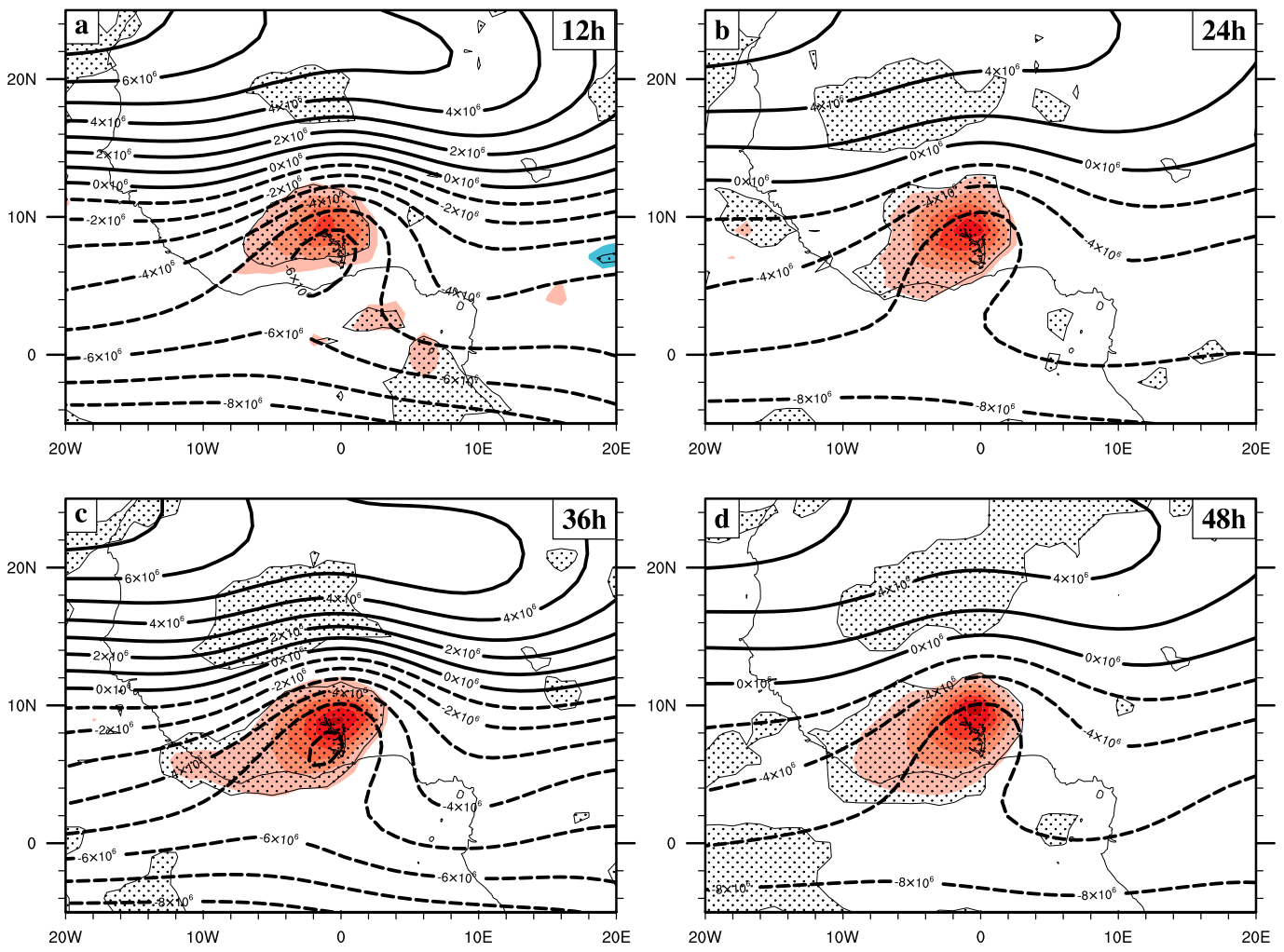

Precipitation Difference $\left(\mathrm{mm}[6 \mathrm{~h}]^{-1}\right)$

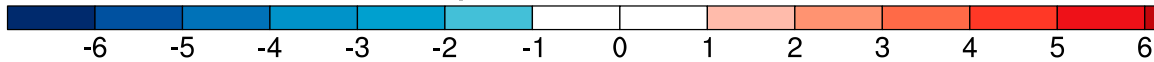

FIG. 3. Wave-centered composite of 2011-13 high SD forecast streamline anomaly (contour) and precipitation rate differences between composite high and low subsets [mm $(6 \mathrm{~h})^{-1}$, color fill] for (a) 12-, (b) $24-$-, (c) 36-, and (d) 48-h forecasts. Stippling indicates locations where the differences are statistically different at the $95 \%$ confidence interval using bootstrap resampling. Composite center is at $9^{\circ} \mathrm{N}, 0^{\circ}$. Geographic background is for illustrative purposes only.

differences in the AEW trough (Fig. 3a). The difference is maximized $1^{\circ}$ west of the trough center $\left[4.45 \mathrm{~mm}(6 \mathrm{~h})^{-1}\right.$ higher precipitation rate, a $97 \%$ increase over the low SD precipitation rate], with differences $>3 \mathrm{~mm}(6 \mathrm{~h})^{-1}$ located at the trough center. Over time, differences increase within the AEW trough center. At $24 \mathrm{~h}$, the high SD forecasts have $5.87 \mathrm{~mm}(6 \mathrm{~h})^{-1}$ higher precipitation rates (a $95 \%$ increase) located $1^{\circ}$ west of the AEW trough center with differences greater than $4 \mathrm{~mm}(6 \mathrm{~h})^{-1}$ within the center (Fig. 3b). By $36 \mathrm{~h}$, and continuing through $48 \mathrm{~h}$, the maximum differences [6.35 and $6.48 \mathrm{~mm}(6 \mathrm{~h})^{-1}$; a $169 \%$ and $306 \%$ increase from the low SD precipitation rates, respectively] move into the trough center (Figs. 3c,d). This suggests that the high SD cases are associated with higher precipitation rates within the AEW trough, which in turn, could favor larger AEW intensity variability.

If uncertainty in diabatic heating is important for AEW predictability, higher precipitation rates are likely not a sufficient condition to generate AEW intensity SD differences. Larger uncertainty in AEW intensity likely requires higher precipitation variability, which would allow for higher variability in convectively generated PV. To address this, the high and low SD subset precipitation rate ensemble SD are also computed in a wave-relative framework using the same methodology employed above. Composite differences of precipitation rate SD indicate the high SD cases are characterized by greater precipitation variability (Fig. 4). At $12 \mathrm{~h}$, the high SD forecasts are associated with greater precipitation rate variability to the west of and within the AEW trough center, with a maximum difference of $4.40 \mathrm{~mm}(6 \mathrm{~h})^{-1}$ (a $99 \%$ increase from the low SD precipitation rate SD) located at the trough center (Fig. 4a). These differences move into the AEW and increase in amplitude to $7.47 \mathrm{~mm}(6 \mathrm{~h})^{-1}$ (a $295 \%$ change from the low SD precipitation SD) by $48 \mathrm{~h}$ (Figs. 4b-d). These results support the validation of the hypothesis that AEW intensity SD is associated with and modulated by differences in the moisture availability and subsequent differences in convective features. 

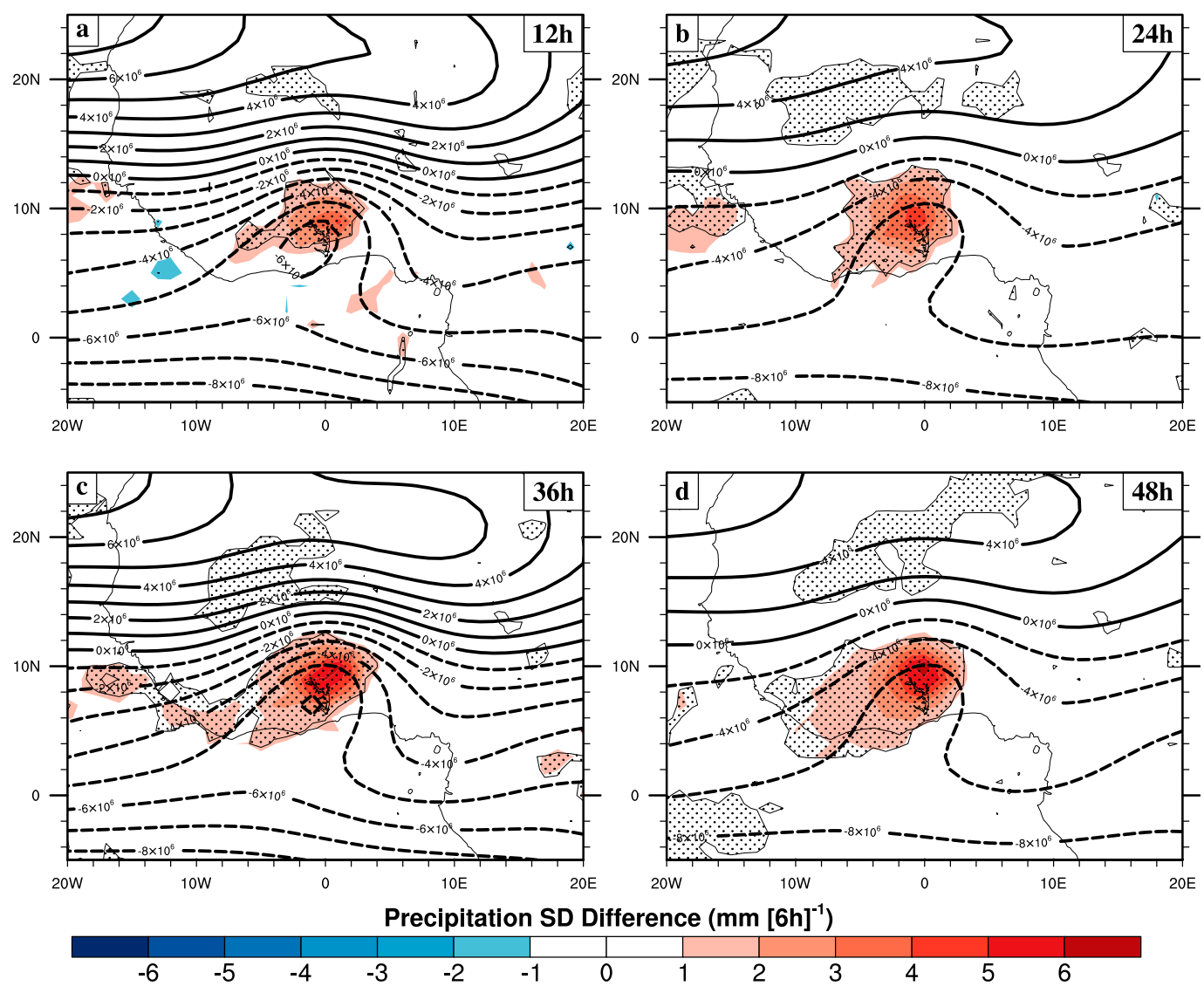

FIG. 4. Wave-centered composite of 2011-13 high SD forecast streamline anomaly (contour) and precipitation rate standard deviation differences between composite high and low subsets [mm (6h) ${ }^{-1}$, color fill] for (a) 12-, (b) 24-, (c) 36-, and (d) 48-h forecasts. Stippling indicates locations where the differences are statistically different at the $95 \%$ confidence interval using bootstrap resampling. Composite center is at $9^{\circ} \mathrm{N}, 0^{\circ}$. Geographic background is for illustrative purposes only.

While the focus thus far has been on diabatic processes, AEWs can also intensify through other means including barotropic and baroclinic instabilities associated with the AEJ. As a consequence, one might hypothesize that a more barotropically and/or baroclinically unstable AEJ would lead to higher AEW intensification rates and hence lower predictability. The hypothesis that AEWs are less predictable in more barotropically unstable environments is assessed by comparing the mean minimum value of $-\partial^{2}[u] / \partial y^{2}$ between the high and low SD cases within the 0 -h control member forecast; this quantity is used to evaluate the background horizontal shear, where $u$ is the 700-hPa zonal wind, and the [] denote a zonal average within $\pm 20^{\circ}$ longitude of each individual AEW trough center between $5^{\circ}$ and $25^{\circ} \mathrm{N}$. Calculating this quantity over a large longitude range is meant to minimize the effects of the AEW trough on the AEJ. This approach is similar to what is used in Thorncroft and Rowell (1998) to measure the instability of the AEJ within a general circulation model. The high SD cases are associated with a significantly more negative $-\partial^{2}[u] / \partial y^{2}$ than the low SD subset (Table 1), and suggests the hypothesis that high SD forecasts tend to occur when the AEJ is more barotropically unstable could also be valid.

Similarly, the hypothesis that AEWs are less predictable when the AEJ promotes an increase in baroclinic instabilities is evaluated using an area-averaged $1000-700-\mathrm{hPa}$ shear magnitude within $10^{\circ}-20^{\circ} \mathrm{N}$ and $\pm 20^{\circ}$ longitude of the AEW trough center. Unlike the barotropic instability, the high SD cases are not associated with a significantly different baroclinic instability environment, suggesting this hypothesis is not valid.

The results presented thus far suggest that AEW intensity SD is associated with differences in several factors; however, it is possible that the AEW intensity predictability also depends on the AEW location and intensity. This possibility is assessed by comparing the mean position and intensity differences between the high and low SD subsets. On average, the low SD subset tends to occur $4^{\circ}$ farther west than the high SD subset; 
TABLE 1. Mean values of the minimum $-\partial^{2}[u] / \partial y^{2}$ (units: $1 \times 10^{-11} \mathrm{~s}^{-1} \mathrm{~m}^{-1}$ ) and area-averaged 1000-700-hPa shear (units: $\mathrm{m} \mathrm{s}$ ). The bootstrap columns denotes the percentile in which the difference between the high SD and respective subsets falls within a 10000 iteration bootstrap resampling distribution where the italic denotes instances where the differences between the two subsets is statistically significant at the $95 \%$ confidence interval.

\begin{tabular}{lccccc}
\hline \hline \multicolumn{1}{c}{ Variable } & High SD & Low SD & Low SD bootstrap & Analog & Analog bootstrap \\
\hline$u_{y y}$ & -8.28 & -7.12 & 1.69 & -8.10 & 37.59 \\
$1000-700-$ hPa shear & 10.39 & 10.33 & 57.46 & 10.81 & 10.87 \\
\hline
\end{tabular}

however, these results are not statistically significant (Fig. 5a). The lack of significant differences could suggest that regional differences in convection may not impact AEW intensity SD. AEW intensity SD also appears to be a function of the AEW intensity itself. The high SD subset is significantly more intense than the low SD subset at $0 \mathrm{~h}\left(1.09 \times 10^{-5} \mathrm{~s}^{-1}\right.$ vs $0.84 \times 10^{-5} \mathrm{~s}^{-1}$ respectively; Fig. 5c). The high SD subset intensity continues to increase throughout the forecast, while the low SD cases weaken by $10 \%$. As a consequence, it appears that less predictable AEWs are intensifying over time.

\section{b. High intensity $S D$ versus analog}

One possible explantation for the results shown in section $4 \mathrm{a}$ is that the intensity variability differences are linked with the initial intensity and/or position differences since convective activity over East Africa generally promotes more diabatic heating at $700-\mathrm{hPa}$ than West Africa (e.g., Janiga and Thorncroft 2013). This possibility is evaluated by employing an analog approach whereby each forecast in the high SD subset is matched with a forecast in the bottom 50th percentile in 72-h AEW intensity SD. This process uses a cost function based on intensity and zonal position evolution to identify an analog that is most similar to each high SD forecast. The analog forecast for the $i$ th high SD case is determined by minimizing the cost function:

$$
\begin{aligned}
J(i, k)= & \frac{\sum_{t=0}^{72 \mathrm{~h}}\left(\mathrm{INT}_{\mathrm{high}_{i, t}}-\mathrm{INT}_{\mathrm{bottom}_{k, t}}\right)^{2}}{\sigma_{\Sigma \Delta \mathrm{int}_{\mathrm{all}}(i)}} \\
& +\frac{\sum_{t=0}^{72 \mathrm{~h}}\left(\mathrm{LON}_{\mathrm{high}_{i, t}}-\mathrm{LON}_{\mathrm{bottom}_{k, t}}\right)^{2}}{\sigma_{\Sigma \Delta \operatorname{lon}_{\mathrm{all}}(i)}},
\end{aligned}
$$

where INT (LON) refers to the ensemble-mean AEW intensity (longitude) at lead time $t, k$ is the index for the bottom 50th percentile of 72-h intensity SD cases, and $\sigma_{\Sigma \Delta \text { int all }_{\text {al }}(i)}\left(\sigma_{\Sigma \Delta \operatorname{lon}_{\text {all }}(i)}\right)$ is the SD of $0-72$-h averaged intensity (longitude) differences for the $i$ th high SD forecast. Dividing by the SD normalizes the metrics, which in turn allows these two metrics to be considered together. This approach is similar to Halperin and Torn
(2018), who employed a similar methodology to identify analog TC intensity forecasts based on maximum wind speed and shear magnitude. For each high SD forecast, the bottom 50th percentile forecast with the lowest cost function value is deemed the best analog for that forecast. Using this analog approach removes the tendency for lower SD systems to occur farther west (Fig. 5b). Furthermore, the difference in 0 -h intensity between the high SD and lower $\mathrm{SD}$ analog forecasts is also negligible from 0 to $48 \mathrm{~h}$ (Fig. 5d). As a consequence, using this analog approach allows for a more homogenous comparison of the environment between the high and low predictability cases.

Comparing the high SD cases to the analog fields indicates increasing differences in $\mathrm{RH}$ with time. At 0 hour, the $700-\mathrm{hPa} \mathrm{RH}$ within $500 \mathrm{~km}$ of the AEW for the high SD cases are similar to the analog cases (Fig. 6a). Beyond that time, the high SD RH remains near $75 \%$ while the analog cases decreases to $66 \%$. The differences in the near-wave environment are most pronounced to the northwest of the AEW trough at 0 -h (Fig. 7a). Over time, these difference become larger and rotate counterclockwise to the west of the AEW, such that the high SD subset is associated with a $10 \%-25 \%$ higher $700-\mathrm{hPa} \mathrm{RH}$ by $48 \mathrm{~h}$ (Figs. 7b-e). This RH difference suggests that the high SD forecasts may not be advecting as much drier Saharan air ahead of the AEW relative to their analog counterparts. As a consequence, the increased moisture in the high SD subset suggests it is easier to initiate and sustain convection in these cases.

Given the large RH differences to the northwest of the AEW in the first $12 \mathrm{~h}$, it is not surprising that the 12 -h precipitation differences are also maximized at this location (Fig. 8a). As the forecast evolves and the high SD AEWs move into this higher RH environment, precipitation rate differences increase within the AEW trough, such that the high SD cases experience a $5.62 \mathrm{~mm}(6 \mathrm{~h})^{-1}$ higher precipitation rate (a $125 \%$ increase) within the trough center by $36 \mathrm{~h}$ (Fig. $8 \mathrm{c}$ ). By $48 \mathrm{~h}$, the ensemble-mean precipitation rate in the high SD cases increases further and remains in the trough center (Fig. 8d), with this pattern continuing for the remainder of the forecast period (not shown).

The regions with statistically significant larger precipitation rates in the high SD cases are also characterized by higher precipitation SD. By $24 \mathrm{~h}$, precipitation SD in 
High SD vs Low SD
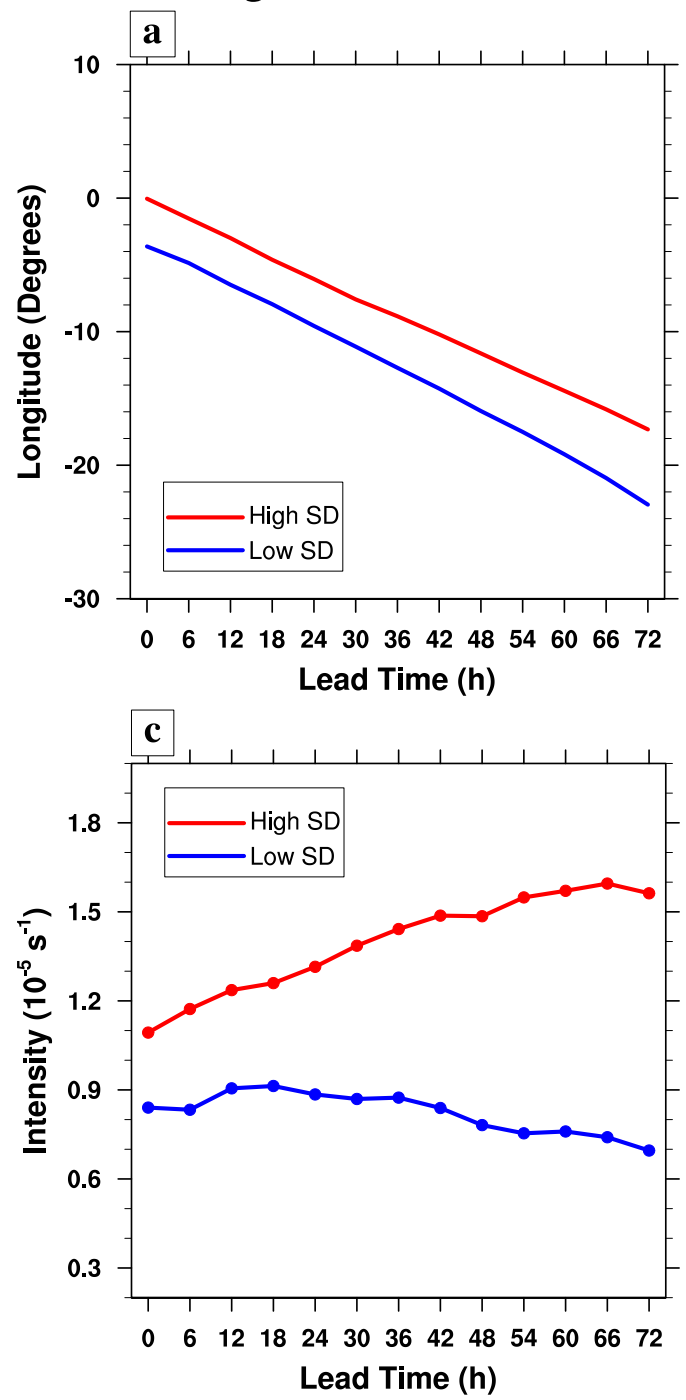

High SD vs Analog
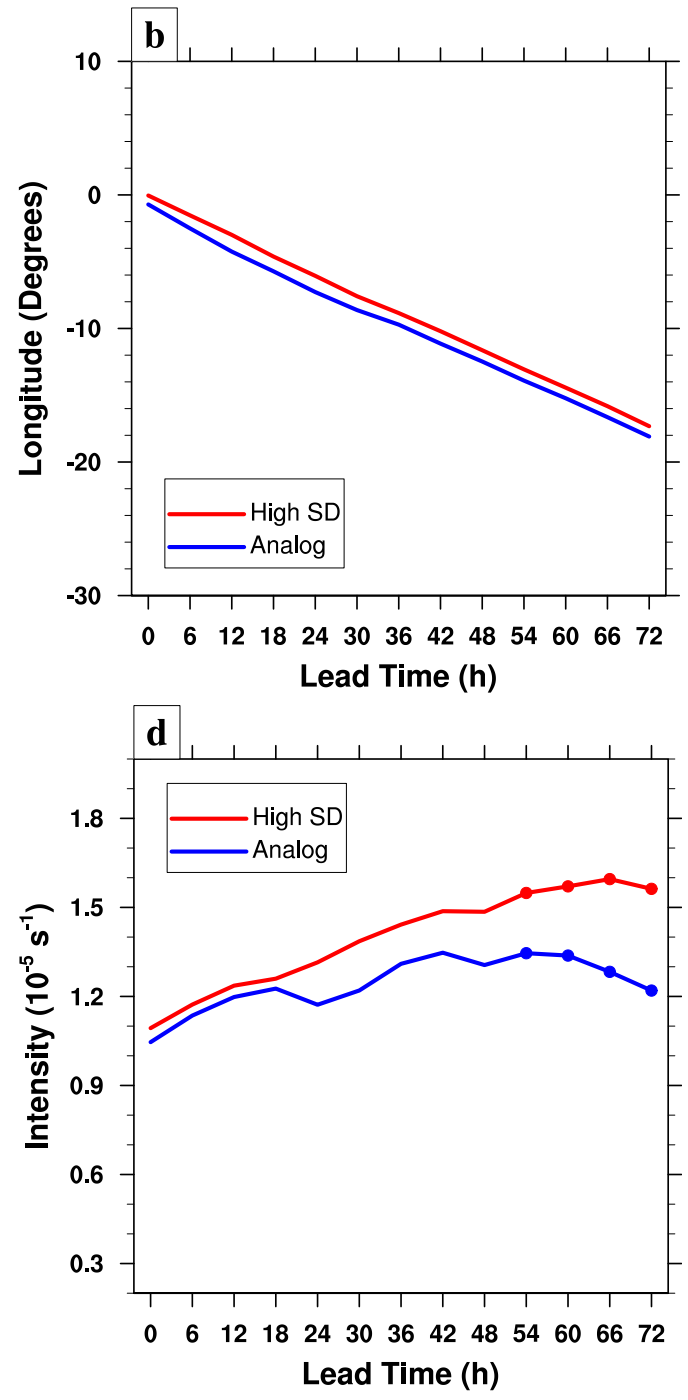

FIG. 5. Composite AEW (a) longitude and (c) intensity for cases in the high (red lines) and low (blue lines) SD subsets with a comparison between composite AEW (b) longitude and (d) intensity for cases in the high SD (red lines) and analog (blue lines) subsets. Dots indicated times when the subsets are statistically different at the $95 \%$ confidence interval using bootstrap resampling.

the high SD forecasts are $2.8 \mathrm{~mm}(6 \mathrm{~h})^{-1}$ higher than in the analog subset (a $50 \%$ increase; Fig. $9 \mathrm{~b}$ ) in the same region of statistically larger 24 -h ensemble-mean precipitation (cf. Fig. 8b). The precipitation SD differences continue to increase within the AEW trough, such that the high SD cases have $6.87 \mathrm{~mm}(6 \mathrm{~h})^{-1}$ higher SD (a $219 \%$ increase) by $48 \mathrm{~h}$ (Fig. 9d). These results suggest that differences in the downstream convective environment plays a crucial role in enhancing convection variability for this period, and this result is not solely related to position differences.

While the high SD cases are characterized by significant differences in thermodynamic environment relative to the analogs, these two sets of cases exhibit similar AEJ properties. Both the baroclinic and barotropic instability show statistically indistinguishable differences between the high SD and analog subsets (Table 1). This suggests that environments with larger barotropic instabilities are associated with stronger AEWs, but are not necessarily associated with creating higher AEW intensity SD. These results suggest the moisture/convection hypothesis is the only valid hypothesis associated with AEW intensity variability.

\section{c. Case study analysis}

The analysis so far has focused on using subset mean values to determine if large-scale processes contribute to 

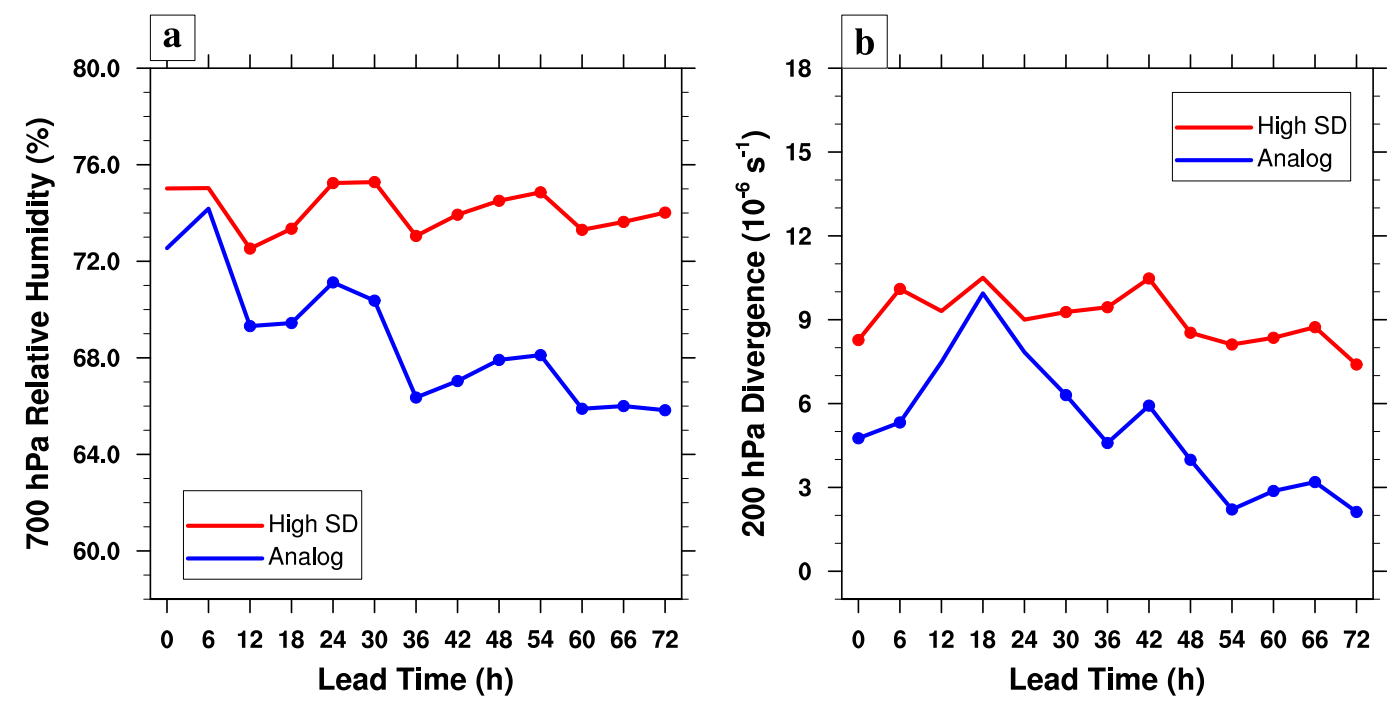

FIG. 6. Composite (a) 700-hPa relative humidity and (b) 200-hPa divergence averaged within $500 \mathrm{~km}$ of the AEW trough center for cases in the high SD (red) and analog (blue) subsets. Dots indicated times when the two subsets are statistically different at the $95 \%$ confidence interval using bootstrap resampling.

AEW intensity predictability. However, this approach can mask some of the case-to-case variability in the processes that impact AEW predictability, which Komaromi and Majumdar (2015) noted exist in AEW intensity forecasts. As a consequence, it is worthwhile to determine whether an individual high SD case is characterized by larger downstream moisture and more extensive convection relative to its individual analog forecast.

The focus of this subsection is AEW-201234 in the Elless and Torn (2018) AEW database. This wave was first identified over Mali $\left(12.1^{\circ} \mathrm{N}, 6.0^{\circ} \mathrm{W}\right)$ at $1200 \mathrm{UTC}$ 4 September 2012. This AEW intensified quickly over West Africa, reaching a maximum intensity of $1.80 \times$ $10^{-5} \mathrm{~s}^{-1}$ at 1200 UTC 7 September 2012 just before moving into the Atlantic Ocean. Seventy-two hours later, the National Hurricane Center (NHC) would classify this disturbance as a tropical depression that would eventually strengthen into Hurricane Nadine (Brown 2013). The focus for the remainder of this subsection is the ECMWF EPS forecast initialized at 0000 UTC 6 September 2012, when AEW-201234 is located over southeastern Senegal, and is $36 \mathrm{~h}$ before peak intensity, and $108 \mathrm{~h}$ before the system is declared a tropical depression. This particular forecast represents the fifth highest 72-h intensity SD found over the 2011-13 period.

Similar to the mean over all cases, AEW-201234 is associated with higher water vapor compared to its individual analog forecast (AEW-201306; forecast initialized at 0000 UTC 9 August 2013). The high SD case is associated with significantly higher $700-\mathrm{hPa} \mathrm{RH}$ to the northwest of the AEW trough center at $0 \mathrm{~h}$ (Fig. 10a). Over the next $48 \mathrm{~h}$, the largest $\mathrm{RH}$ differences move into the trough center (Figs. 10b-e), which suggests that AEW-201234 moves into a more convectively favored environment relative to AEW-201306. Similar to subset mean, differences in precipitation variability generally occur in the vicinity of significant RH differences. At $12 \mathrm{~h}$, positive precipitation SD differences are mainly located downstream of the AEW trough (Fig. 11a), suggesting that the higher SD case is characterized by greater convective variability downstream. By 36 and $48 \mathrm{~h}$, the precipitation SD differences are concentrated at the AEW trough center (Figs. 11c,d), which may suggest higher variability in diabatically generated PV, and hence higher AEW intensity SD. These results further validate the hypothesis that initial moisture availability and subsequent convection are the key factors that determine AEW intensity predictability.

\section{Spatial and temporal impacts on AEW intensity SD}

The results from the previous section are similar to the findings in Komaromi and Majumdar (2015), which highlight the large-scale moisture availability can influence the AEW intensity forecast predictability by modulating convection in the forecast. However, the question of what drives these moisture differences still remains. Previous studies have highlighted two significant controls on the large-scale moisture availability/ convective activity over Africa: 1) types of convective activity being favored in different regions across Africa (e.g., Janiga and Thorncroft 2013), and 2) moisture variability due to convectively coupled equatorial waves 

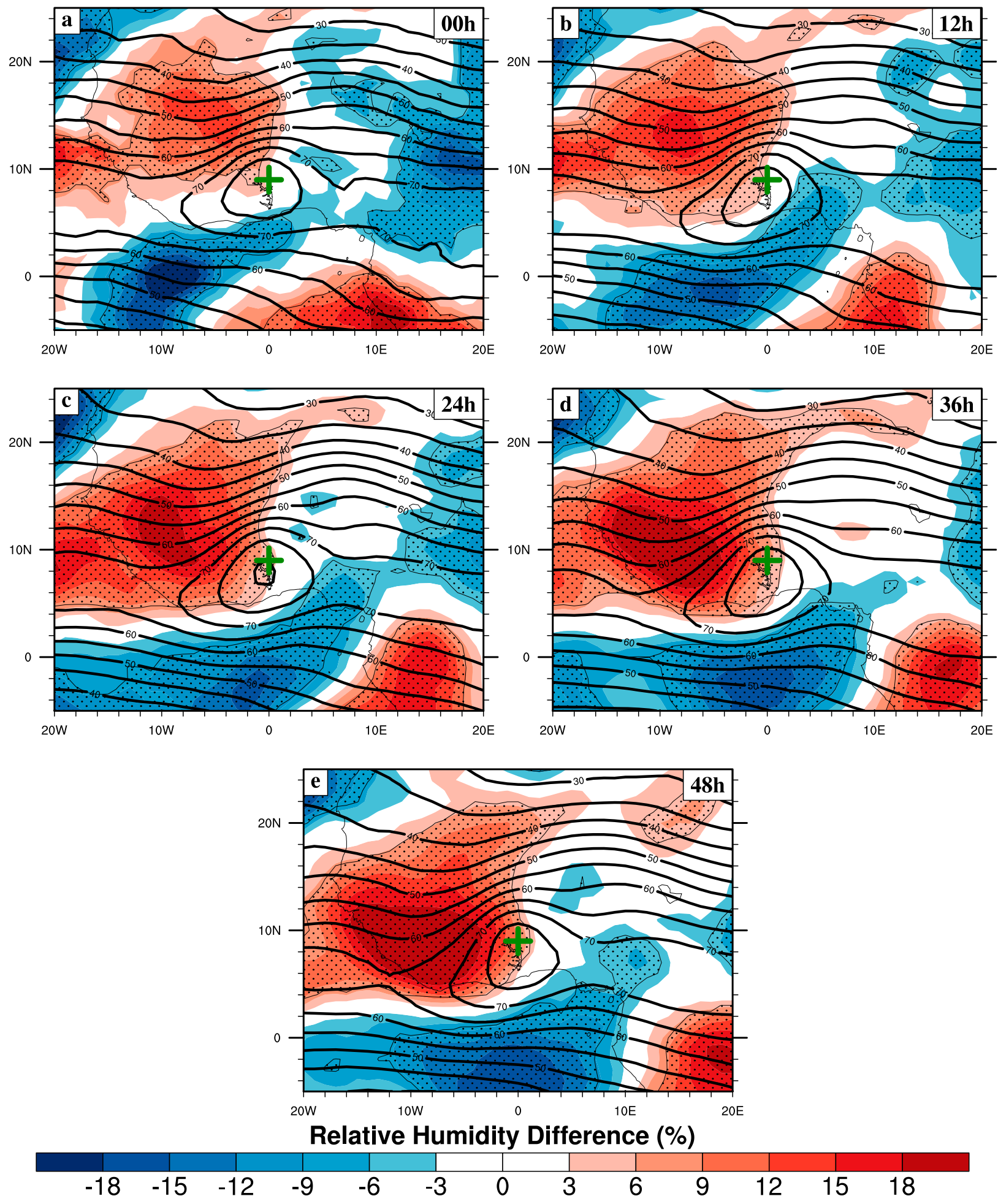

FIG. 7. Wave-centered composite differences in 700-hPa relative humidity between composite high SD and analog subsets (\%, color fill) at (a) 0 , (b) 12, (c) 24, (d) 36, and (e) $48 \mathrm{~h}$. The contours denote the mean 700-hPa RH for all cases. Stippling indicates locations where the differences are statistically different at the $95 \%$ confidence interval using bootstrap resampling. Composite center is at $9^{\circ} \mathrm{N}, 0^{\circ}$, denoted by the crosses. Geographic background is for illustrative purposes only. 

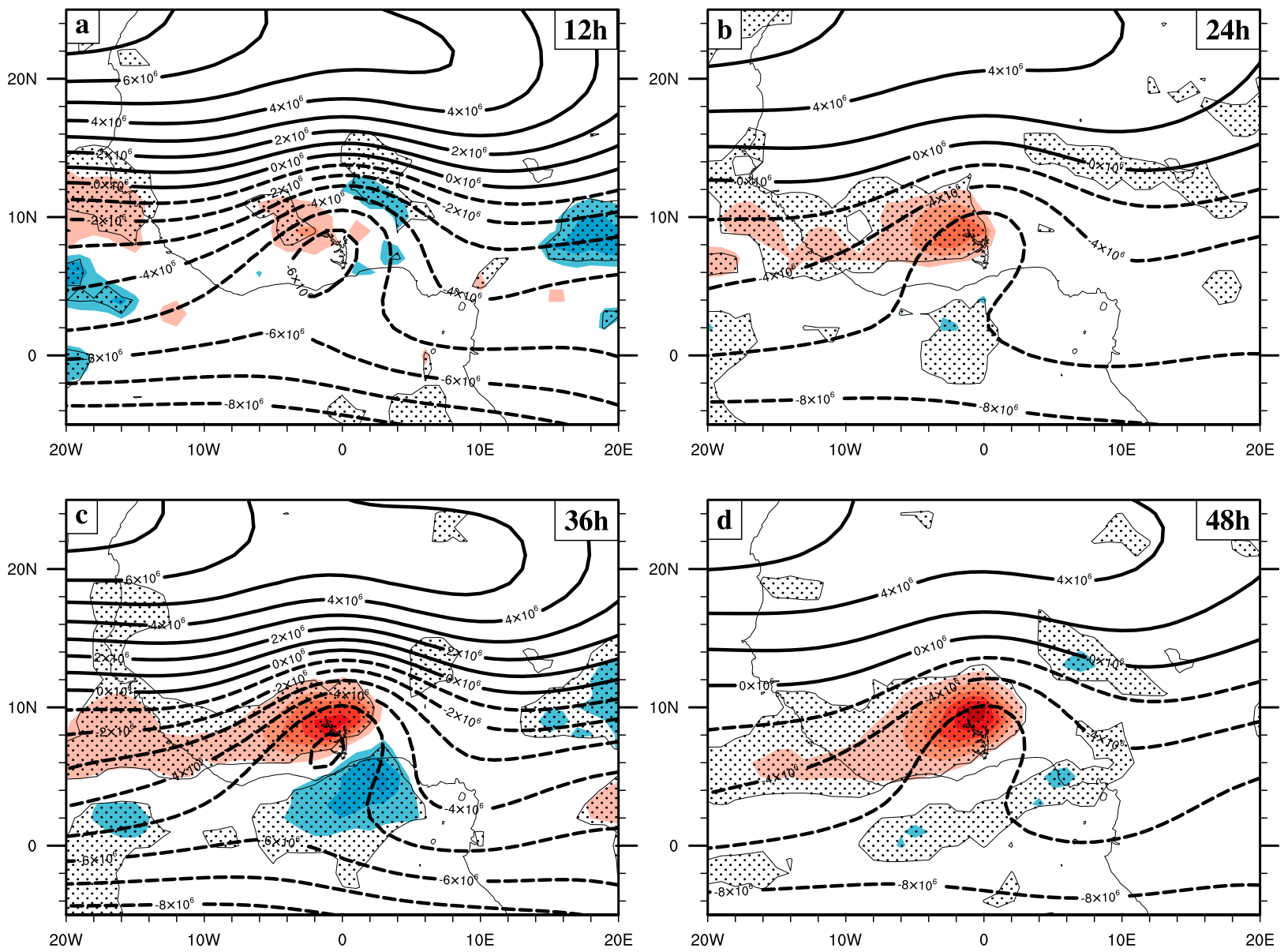

Precipitation Difference $\left(\mathrm{mm}[6 \mathrm{~h}]^{-1}\right)$

\begin{tabular}{llllllllllllll}
\hline & -6 & -5 & -4 & -3 & -2 & -1 & 0 & 1 & 2 & 3 & 4 & 5 & 6
\end{tabular}

FIG. 8. Wave-centered composite of 2011-13 700-hPa high SD forecast streamline anomaly (contour) and precipitation rate differences between composite high SD and analog subsets [mm (6 h) $)^{-1}$, color fill] for (a) 12-, (b) 24-, (c) 36-, and (d) 48-h forecasts. Stippling indicates locations where the differences are statistically different at the $95 \%$ confidence interval using bootstrap resampling. Composite center is at $9^{\circ} \mathrm{N}, 0^{\circ}$. Geographic background is for illustrative purposes only.

(e.g., Ventrice et al. 2011; Ventrice and Thorncroft 2013). As a consequence, these factors could be ultimately be responsible for determining AEW intensity predictability; therefore, this section evaluates the hypothesis that a certain region of Africa and/or phases of convectively coupled equatorial waves will favor higher AEW intensity SD.

\section{a. Regional differences}

Diabatic heating rates derived by Janiga and Thorncroft (2013) highlight three distinct regions in subSaharan Africa. Recall that the diabatic heating profile over West Africa and the Atlantic Ocean $\left(40^{\circ}-10^{\circ} \mathrm{W}\right)$ is positive throughout the entire troposphere, which is indicative of deep convection, while central Africa $\left(10^{\circ} \mathrm{W}-4^{\circ} \mathrm{E}\right)$ lacks deep moisture and therefore has lower diabatic heating rates. Finally, East Africa $\left(4^{\circ}-40^{\circ} \mathrm{E}\right)$ is characterized by a "top-heavy" diabatic heating profile, which suggests a greater fraction of stratiform precipitation, with warming above $700 \mathrm{hPa}$ and near-zero or diabatic cooling occurring below that level. To assess the impact of these regional latent heating differences, forecasts are binned into "west," "central," and "east" subsets based on the 0-h position and compared against all forecasts.

Similar to the results in the previous section, there are few regional differences in AEW intensity SD (Fig. 12a). The intensity SD for all regions are indistinguishable from the all-case mean at all lead times, suggesting that regional differences are not a major influence on intensity $\mathrm{SD}$ for this period. The results from the previous section suggest that higher AEW intensity SD is associated with 

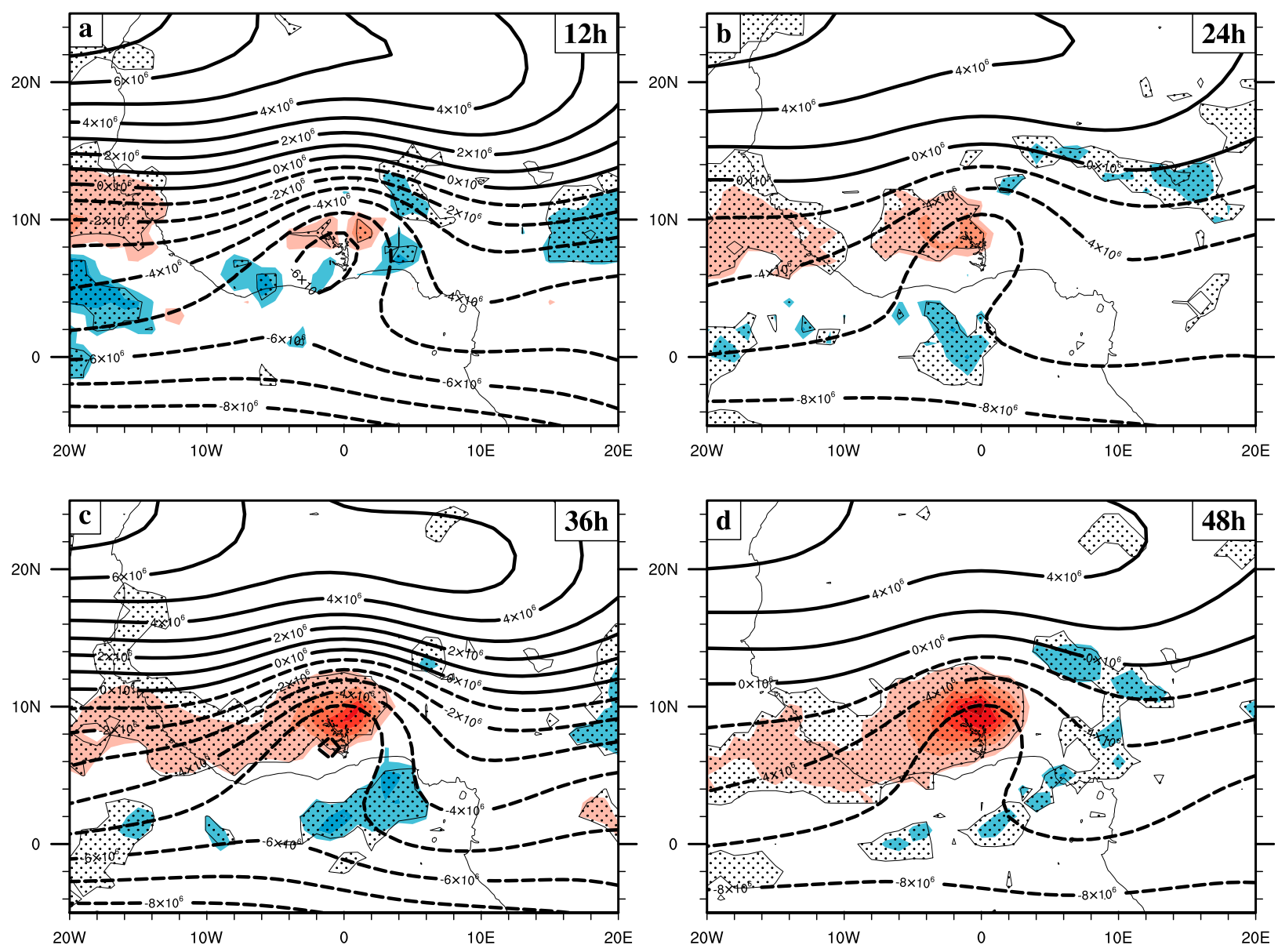

Precipitation SD Difference $\left(\mathrm{mm}[6 \mathrm{~h}]^{-1}\right)$

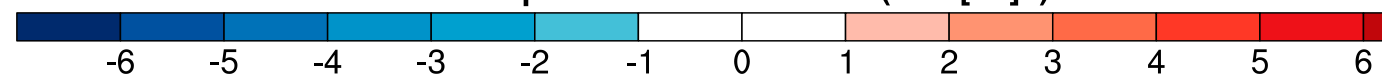

FIG. 9. Wave-centered composite of 2011-13 700-hPa high SD forecast streamline anomaly (contour) and precipitation rate standard deviation differences between composite high SD and analog subsets [mm (6 h) ${ }^{-1}$, color fill] for (a) 12-, (b) 24-, (c) 36-, and (d) 48-h forecasts. Stippling indicates locations where the differences are statistically different at the $95 \%$ confidence interval using bootstrap resampling. Composite center is at $9^{\circ} \mathrm{N}, 0^{\circ}$. Geographic background is for illustrative purposes only.

higher precipitation SD; therefore, the lack of AEW intensity SD differences could be related to the lack of differences in precipitation SD. To test this hypothesis, wave-centered composites of precipitation SD are created for each regional subset as well as a mean of all cases using the previously described method. Composite differences are created by subtracting the 60 -h all-case mean from each regional subset; the 60 -h differences are also spatially representative of differences for other lead times (not shown). A similar precipitation SD pattern exists throughout all regions (Figs. 12b-d), with the west subset associated with a slight decrease in precipitation $\mathrm{SD}\left[-1.89 \mathrm{~mm}(6 \mathrm{~h})^{-1}\right]$ and a slight increase $\left[0.62 \mathrm{~mm}(6 \mathrm{~h})^{-1}\right]$ for the east region. Given these differences are small in comparison to the differences between the high and low SD subsets at $60 \mathrm{~h}\left[17.79 \mathrm{~mm}(6 \mathrm{~h})^{-1}\right]$, it appears that regional differences in precipitation variability are not a significant source of AEW intensity SD.

\section{b. Equatorial waves}

Convectively coupled equatorial waves are associated with large-scale moisture variations over Africa; convectively active phases are generally associated with an increase in large-scale moisture and are often associated with enhanced convection, while the suppressed phases are generally associated with large-scale subsidence resulting in drier conditions (e.g., Fig. 6 in Straub and Kiladis 2003). As a consequence, it is prudent to investigate whether AEW intensity SD is statistically different for various phases of convectively coupled equatorial waves. Convectively coupled equatorial wave phase is identified using the $2.5^{\circ} \times 2.5^{\circ}$ NOAA 

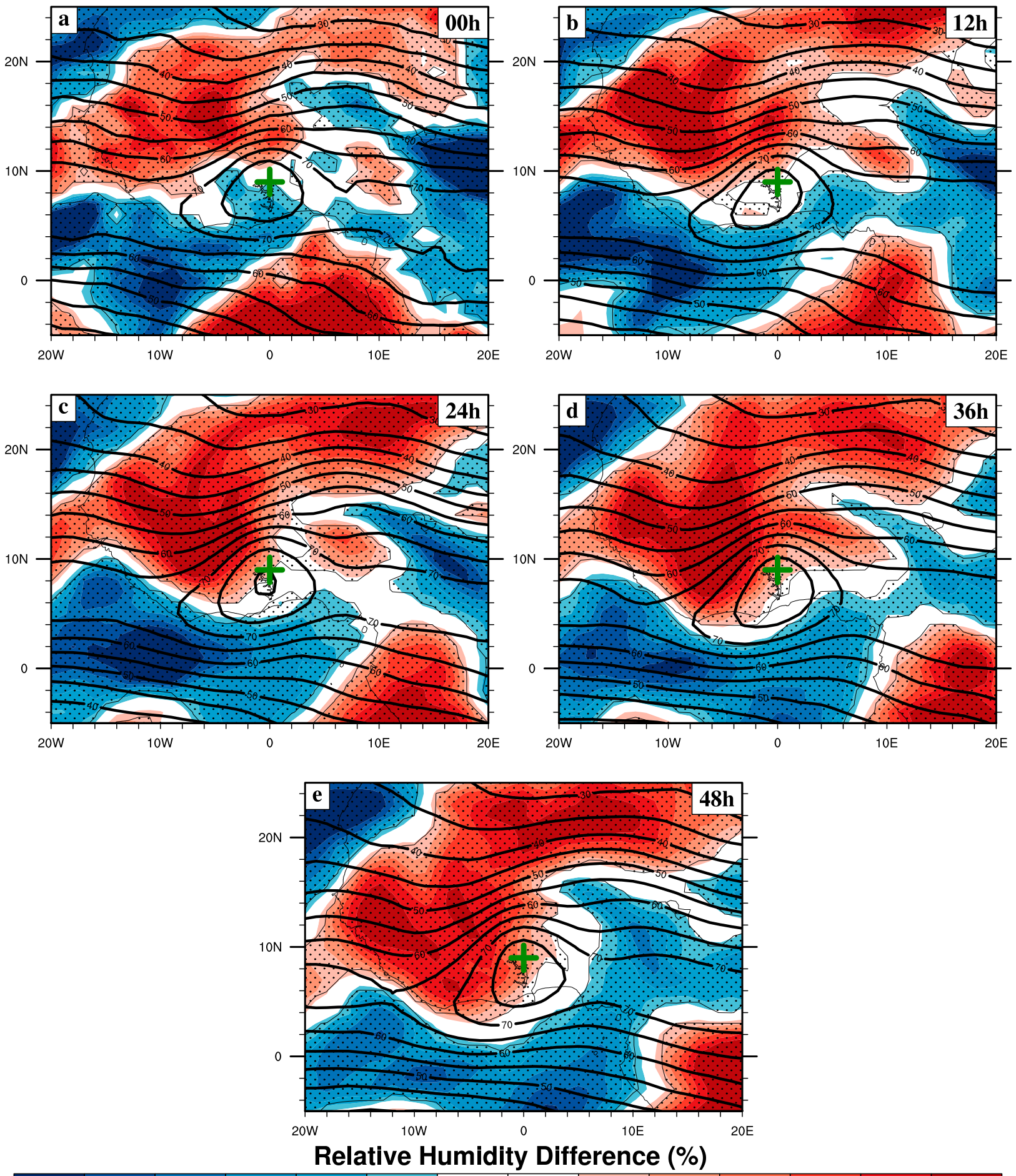

$\begin{array}{lllllllllllll}-36 & -30 & -24 & -18 & -12 & -6 & 0 & 6 & 12 & 18 & 24 & 30 & 36\end{array}$

FIG. 10. Wave-centered composite differences in 700-hPa relative humidity between AEW-201234 (high SD case) and AEW-201306 (analog case; color fill) at (a) 0, (b) 12, (c) 24, (d) 36, and (e) $48 \mathrm{~h}$. The contours denote the mean 700-hPa RH for all cases. Stippling indicates locations where the differences are statistically different at the $95 \%$ confidence interval using bootstrap resampling. Composite center is at $9^{\circ} \mathrm{N}, 0^{\circ}$, denoted by the crosses. Geographic background is for illustrative purposes only. 

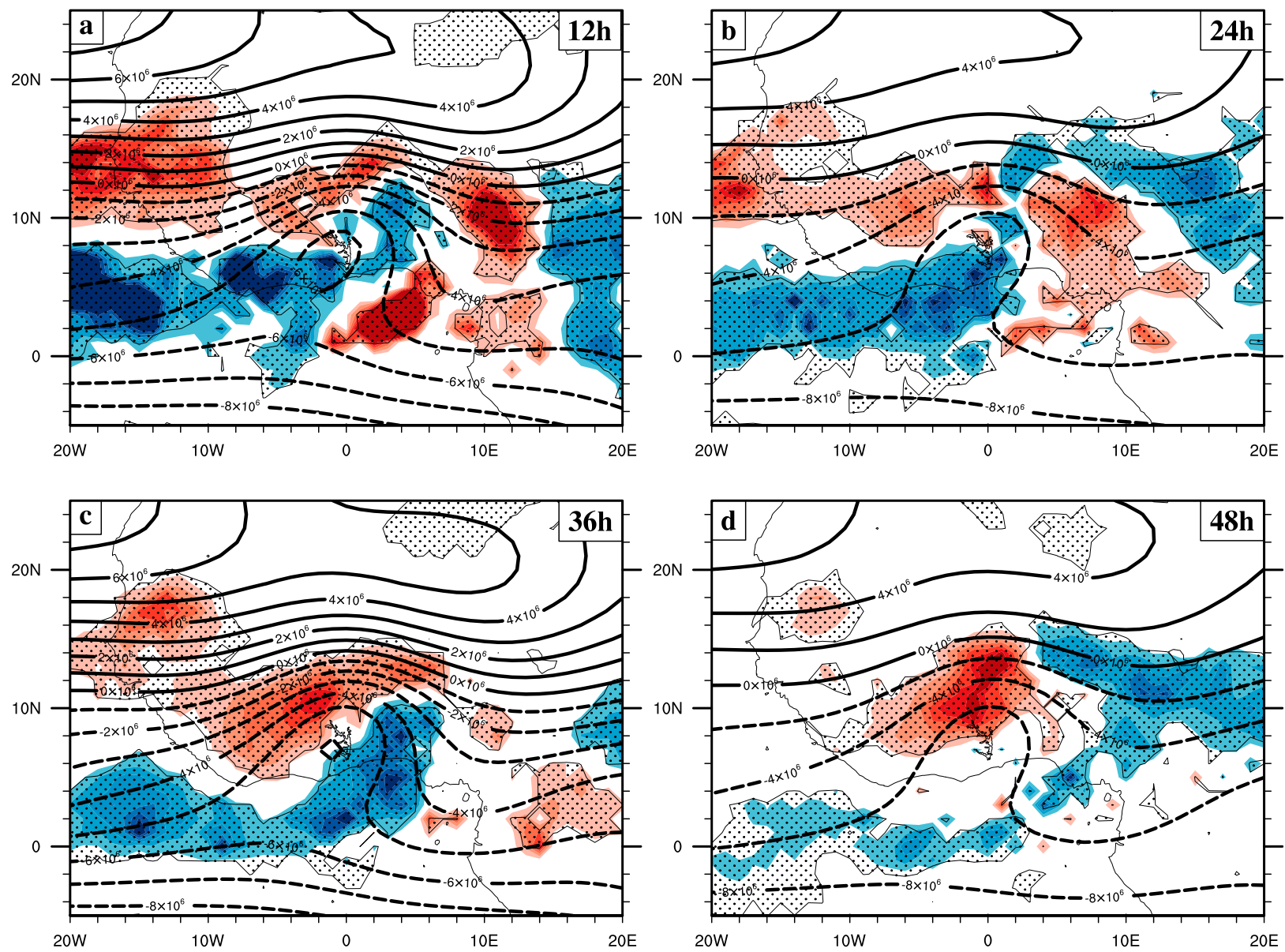

Precipitation SD Difference $\left(\mathrm{mm}[6 \mathrm{~h}]^{-1}\right)$

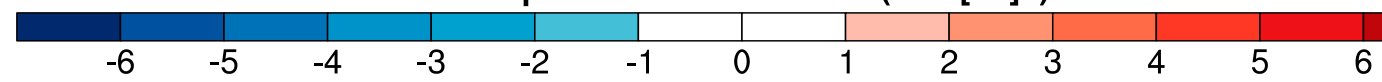

FIG. 11. Wave-centered composite of 2011-13 700-hPa high SD forecast streamline anomaly (contour) and precipitation rate standard deviation differences between AEW-201234 (high SD case) and AEW-201306 (analog case; color fill) for (a) 12-, (b) 24-, (c) 36-, and (d) 48-h forecasts. Stippling indicates locations where the differences are statistically different at the $95 \%$ confidence interval using bootstrap resampling. Composite center is at $9^{\circ} \mathrm{N}, 0^{\circ}$. Geographic background is for illustrative purposes only.

Interpolated outgoing longwave radiation (OLR) dataset (Liebmann and Smith 1996) from 1979 to 2015. These data are averaged in the $5^{\circ}-15^{\circ} \mathrm{N}$ latitude band before filtering spatially and temporally to identify the CCKW, mixed Rossby-gravity wave (MRG), and the equatorial Rossby wave (ER; e.g., Wheeler and Kiladis 1999). The space and time parameters used to identify each equatorial wave are found in Table 2. Local convectively active and suppressed phases of these equatorial waves are then identified by comparing the filtered OLR data and its time derivative, similar to what is done within Yasunaga and Mapes (2012) and van der Linden et al. (2016). Both quantities are first normalized by their SD calculated across all times and all longitudes which allows for a quantitative comparison of terms. The convective phase of the equatorial waves is determined via a phase diagram at $2.5^{\circ} \mathrm{E}$, which represents the mean AEW position. For each equatorial wave, amplitude and phase angle are defined as:

$$
\text { Amplitude }=\sqrt{\text { filteredOLR }{ }^{2}+(d \text { filteredOLR } / d t)^{2}},
$$

$$
\text { Angle }=\arctan \left(\frac{\text { filteredOLR }}{d \text { filteredOLR/dt }}\right) .
$$

Convectively active (suppressed) phases are finally defined as times when the amplitude greater than one SD, and the phase angle is between $225^{\circ}$ and $315^{\circ}\left(45^{\circ}-135^{\circ}\right)$; these times are associated with significant negative (positive) OLR anomalies. Forecasts are then subsetted based on convective phase at time of forecast initialization 

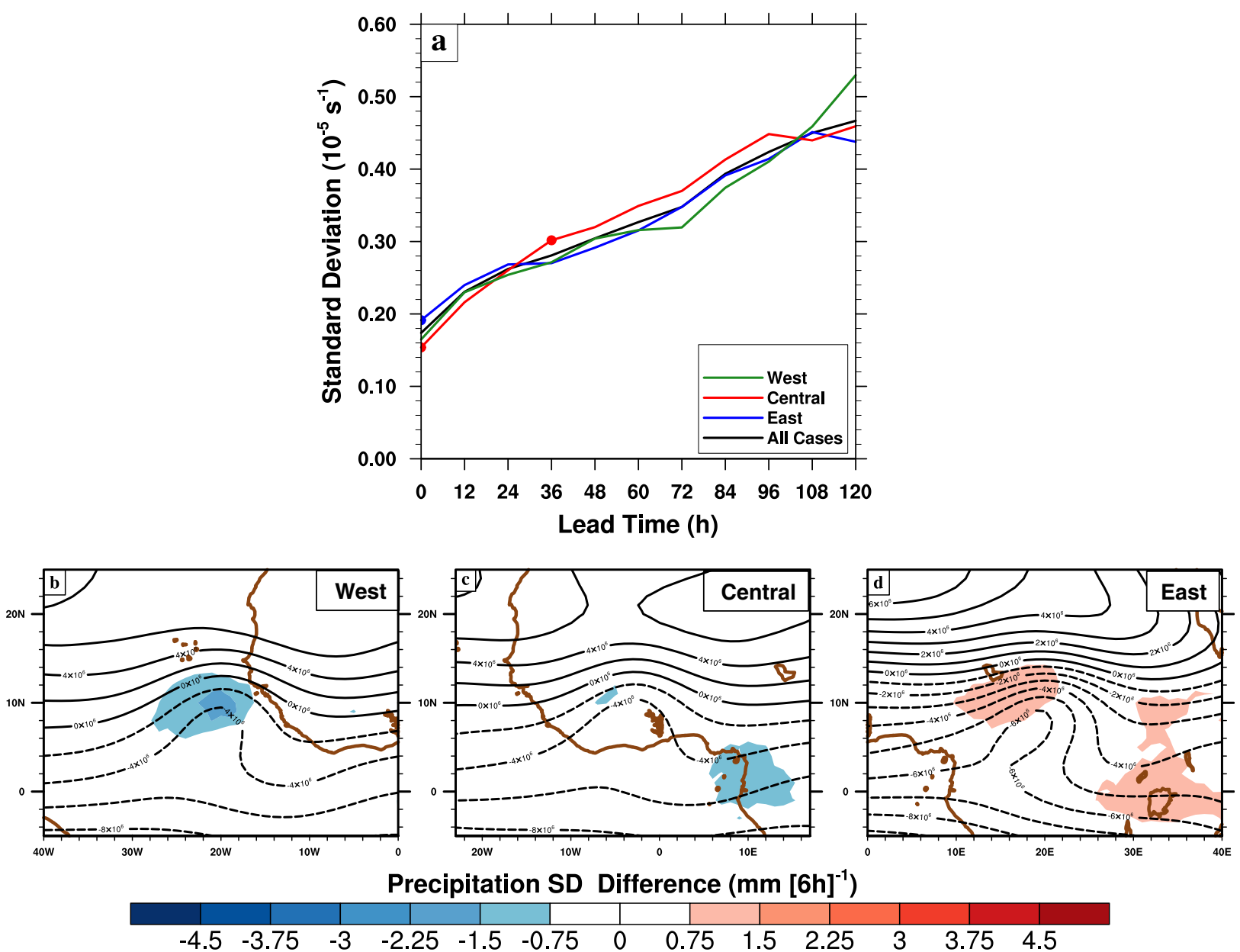

FIG. 12. (a) AEW intensity SD for all cases (black), east subset (blue), central subset (red), and west subset (green) for the 2011-13 period. Dots indicated times when the subsets are statistically different from the all-case value at the $95 \%$ confidence interval using bootstrap resampling. (b)-(d) Wave-centered composite 60-h (b) west, (c) central, and (d) east streamfunction anomaly (contours $\mathrm{s}^{-1}$, dashed contours represent negative value) and precipitation SD differences between the subsets and the all-case mean precipitation SD for the 2011-13 forecast period [color fill; units $\mathrm{mm}(6 \mathrm{~h})^{-1}$ ]. Geographic background is for illustrative purposes only.

to determine if certain phases are more likely to have large AEW intensity SD. MJO-filtered cases were also considered, however, each phase was characterized by too few cases with an amplitude above 1 SD to yield a meaningful result (not shown).

Previous studies noted that the increased moisture/ convection associated with the convectively active phase of equatorial waves tend to produce more frequent and intense AEWs (e.g., Ventrice et al. 2011; Ventrice and Thorncroft 2013). Given that stronger waves also tend to favor large intensity SD (cf. Fig. 5c) and precipitation SD (cf. Fig. 4), a difference in the AEW intensity among the equatorial waves could suggest an increase in AEW intensity and precipitation SD is likely. While the phases of the CCKW during this period do not produce significantly different intensities from climatology, the active phase tends to be associated with stronger intensities than the suppressed phase through the first $60 \mathrm{~h}$ (Fig. 13a), suggesting the convectively active may phase may be associated with an increase in AEW intensity and precipitation SD. A much stronger relationship is seen with the ER phases (Fig. 13c), further suggesting a difference in AEW intensity and precipitation SD could be associated for this wave. The MRG shows a different signal where the convectively suppressed phase tends to produce stronger AEWs (especially later in forecast),

TABLE 2. Spatial and time parameters used to filter for a given equatorial wave.

\begin{tabular}{lccr}
\hline \hline Wave type & Propagation & Wavenumber & Period \\
\hline CCKW & Eastward & $1-14$ & $2.5-20$ \\
MRG & Westward & $1-10$ & $3-10$ \\
ER & Westward & $1-10$ & $9-72$ \\
\hline
\end{tabular}



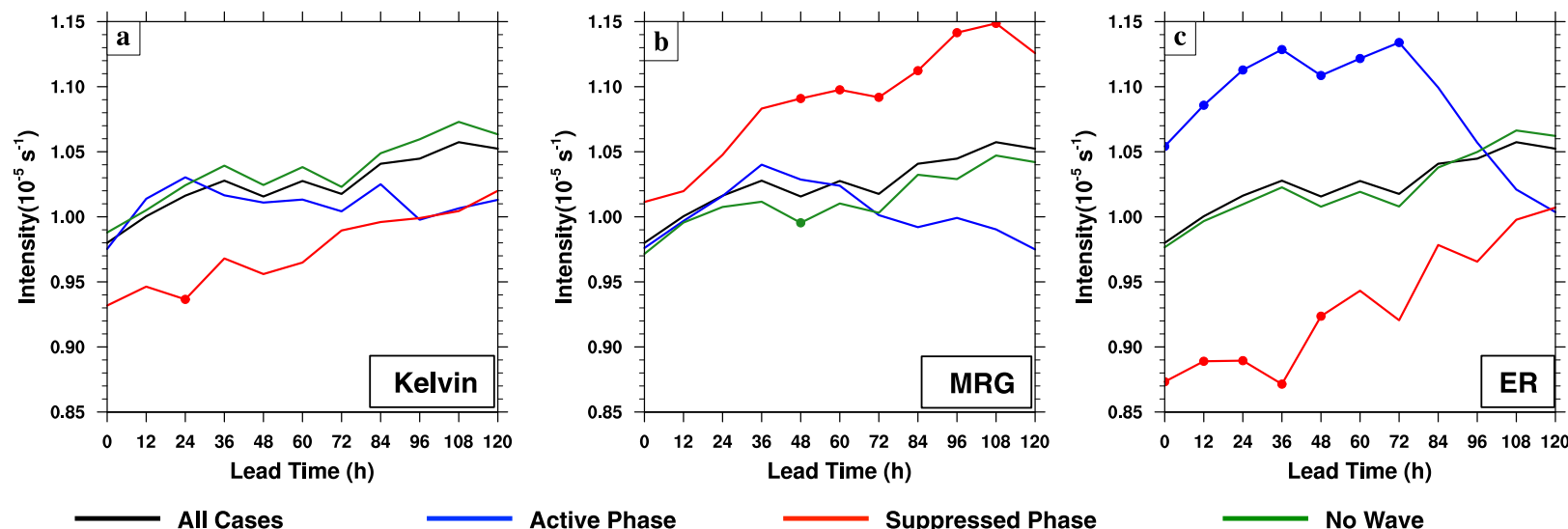

FIG. 13. Average AEW intensity during the convectively active phase (blue), convectively suppressed phase (red), and all remaining cases (green) for (a) Kelvin wave, (b) mixed Rossby-gravity wave, and (c) equatorial Rossby wave during the 2011-13 forecast period. Dots indicated times when the phase values are statistically different from the all-case value (black) at the $95 \%$ confidence interval using bootstrap resampling.

suggesting greater SDs are likely during this phase. One possible reason for this difference is that the MRG period ranges from 3 to 10 days (Table 2), it is possible to have a transition between convectively suppressed and active phases over the African continent in $72 \mathrm{~h}$. As a consequence, cases initialized during the suppressed phase can interact with the increased moisture associated with the active phase.

While the phase intensities suggest a relationship is possible between the convectively coupled equatorial waves and AEW intensity SD, AEW intensity SD appears to be relatively insensitive to convectively coupled equatorial wave phase, with the exception of the MRG waves. While the 0-h AEW intensity SD is similar for the active and suppressed phases of the MRG, the suppressed MRG phase are systematically associated with larger intensity SD beyond $48 \mathrm{~h}$ compared to the all-case mean (Fig. 14b). The transition hypothesis posed above is apparent in wave-centered composite $700-\mathrm{hPa} \mathrm{RH}$ differences between the suppressed and active phases of the MRG (Figs. 15b,e). At $0 \mathrm{~h}$, the forecasts initialized in the suppressed phase are associated with an increase in 700-hPa RH to the northwest of the AEW trough near $15^{\circ} \mathrm{N}$ and $16^{\circ} \mathrm{W}$, similar to the differences observed between the high SD and analog subsets (cf. Fig. 7). By $48 \mathrm{~h}$, the AEW moves toward the 0-h RH differences, which allows the AEW to experience higher RH (Fig. 15e). Similar to the results from the previous section, these results suggest that moving an AEW into a more convectively favorable environment is linked to increasing the AEW intensity SD for these cases. The ER is the only other wave to show significantly significant results as the suppressed phase favoring a reduction in AEW intensity SD during the first $36 \mathrm{~h}$ (Fig. 14c). Comparing the suppressed and active ER phases shows the suppressed phase is associated with significantly less
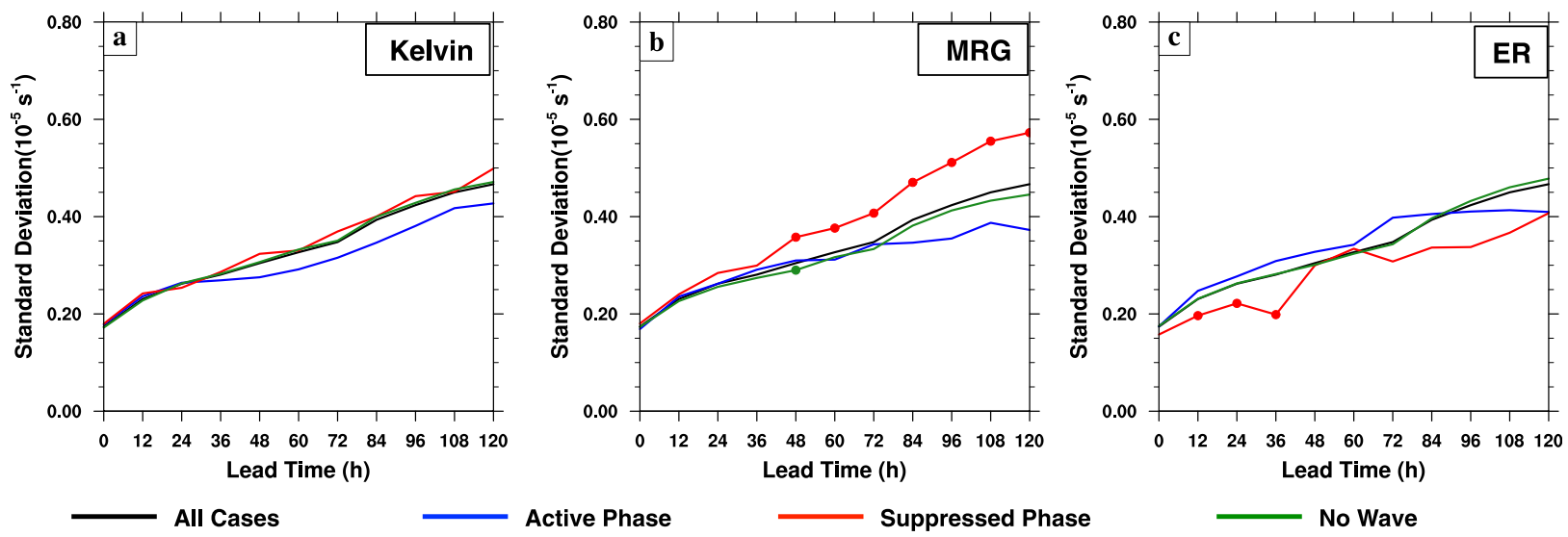

FIG. 14. As in Fig. 13, but for the AEW intensity SD. 


\section{Kelvin}

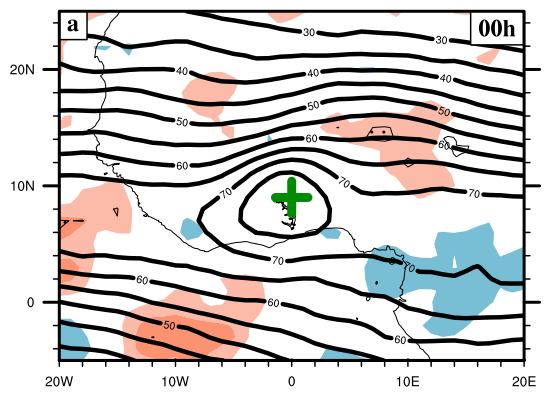

MRG

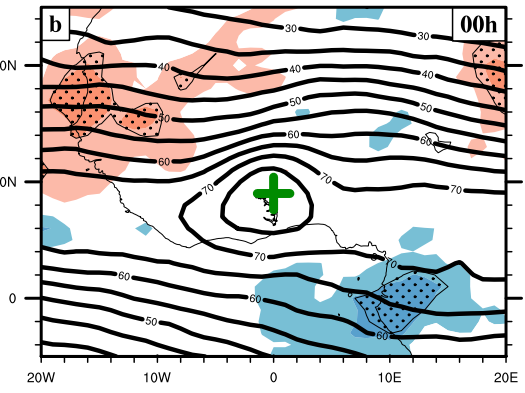

ER

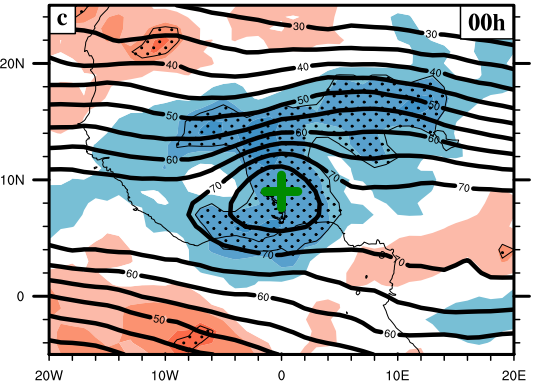

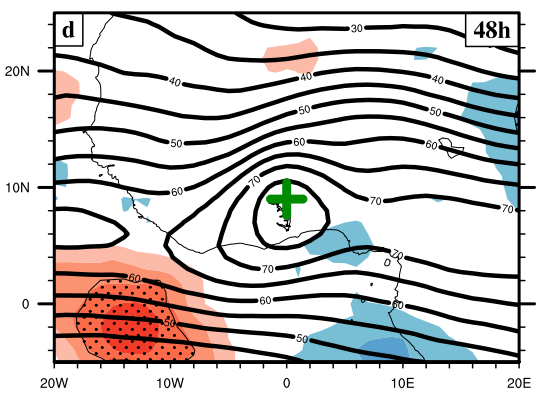
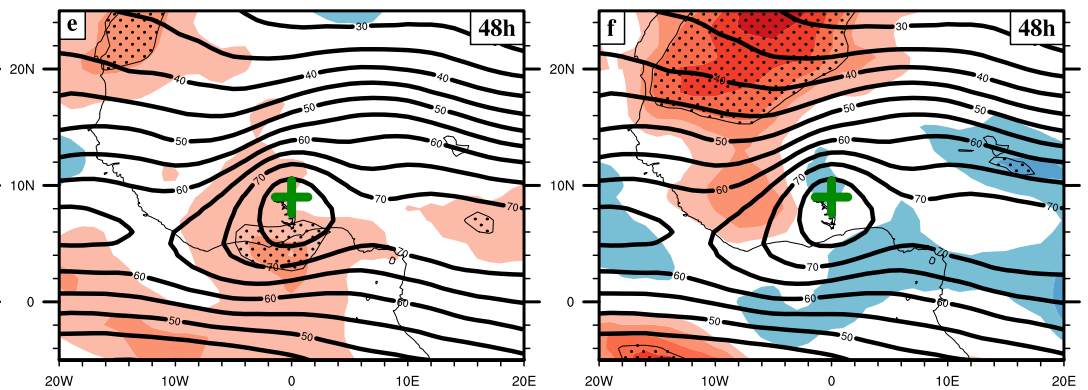

Relative Humidity Difference (\%)

$\begin{array}{lllllllllllll}-18 & -15 & -12 & -9 & -6 & \mid & \mid & \mid & \mid & \mid & \mid & \mid & \mid\end{array}$

FIG. 15. Wave-centered composite of relative humidity for all cases (contour) and 700-hPa relative humidity differences between suppressed and active equatorial wave subsets (\%, color fill) at (a)-(c) 0 and (d)-(f) $48 \mathrm{~h} \mathrm{for} \mathrm{(left)} \mathrm{Kelvin,} \mathrm{(middle)} \mathrm{MRG,} \mathrm{and} \mathrm{(right)} \mathrm{ER}$ waves. Stippling indicates locations where the differences are statistically different at the $95 \%$ confidence interval using bootstrap resampling. Composite center is at $9^{\circ} \mathrm{N}, 0^{\circ}$, denoted by the crosses. Geographic background is for illustrative purposes only.

$\mathrm{RH}$ within the trough center at $0 \mathrm{~h}$ (Fig. 15c). Much like the previous results, it appears the reduced moisture availability is associated with reducing the AEW intensity SD. By $48 \mathrm{~h}$, the AEW is no longer located in the region of lower RH within the AEW trough (Fig. 15f), which can allow for similar convection to occur within the suppressed and active ER phases. The CCKW are generally associated with a similar intensity SD distribution as the all-case mean (Fig. 14a), suggesting that the phase of these waves do not play a significant role in modulating AEW intensity SD. Unlike the ER and MRG waves, the suppressed and active CCKW phases have similar RH in the AEW (Figs. 15a,d), suggesting AEWs experience a similar convective environment in each phase and produce similar AEW intensity SD.

\section{Summary and conclusions}

This study evaluated multiple hypotheses for how the AEW and large-scale environment influence the predictability of AEW intensity forecasts using the ECMWF EPS during 2011-13. Here, wave-centered composites were used to compare the environment of cases characterized by high SD (i.e., low predictability) 72-h AEW intensity forecasts against 72 -h intensity forecasts that were characterized by lower SD and hence are more predictable. AEW intensity forecasts have higher SD in environments characterized by higher water vapor downstream of the AEW at $0 \mathrm{~h}$, which is consistent with the results of Torn (2010) and Komaromi and Majumdar (2015). Over time, the higher initial water vapor content downstream promotes more extensive convection near the AEW. As the AEW moves toward this region, convective differences occur within the AEW trough promoting differences in PV generation within the trough; these convective-scale PV differences can in turn grow upscale and affect the AEW intensity. As a consequence, AEW intensity predictability is likely determined by diabatic processes related to downstream 0 -h moisture availability. Therefore, these results suggests that the paradigm proposed by Durran and Gingrich (2014) and Durran and Weyn (2016) may apply to AEW intensity predictability. By contrast, AEW intensity predictability does not appear to be modulated by the amount of baroclinic or barotropic instability, despite the fact that previous studies have highlighted the importance of this to AEW intensification. These null results further suggest that the 0-h moisture availability is key in determining AEW intensity SD. 
This study also explored hypotheses for how the two biggest controls of moisture over Africa, namely large-scale regional moisture differences and convectively coupled equatorial waves, impact the predictability of AEW intensity forecasts. AEW intensity SD does not appear to be a function of longitude, suggesting the varying regional diabatic heating profiles are not important in this version of the ECMWF EPS. Furthermore, convectively coupled equatorial waves generally do not favor an increase or decrease in AEW intensity SD, with the exception of the suppressed phase of the MRG. This suggests the Kelvin wave processes observed in Ventrice and Thorncroft (2013) do not systematically impact AEW intensity SD, although, an in-depth study on the MRG and ER relationship with AEWs does not exist in the literature. Given the MRG was the only equatorial wave associated with a change in AEW intensity predictability, a more detailed analysis into the relationship between the MRG and AEW should be explored. Furthermore, given largescale moisture variations do not play a major role in creating AEW intensity SD, it appears that smaller-scale features such as a downstream AEW could modulate moisture transport ahead of the second trough (e.g., Brammer and Thorncroft 2015) and/or variations in the advection of dry air ahead of the trough have the potential to impact AEW intensity predictability, and should be areas of focus for future work. Finally, the large-scale features assessed in this study only include a fraction of the possible factors that could affect precipitation and AEW intensity SD. Therefore, further work is also needed to focus on the impacts of such large-scale teleconnection features such as the El Niño-Southern Oscillation (ENSO) and the Atlantic multidecadal oscillation (AMO). These types of future studies would require a stable model configuration over several decades, such as from a reforecast dataset.

Acknowledgments. We thank our colleagues at the University at Albany, Yuan-Ming Cheng and Chris Thorncroft, for providing valuable feedback throughout the completion of this work. We are also grateful to the constructive comments from Will Komaromi and two anonymous reviewers in helping to improve this paper. This work is supported by the National Science Foundation through NSF Awards 1321568 and 1461753.

\section{APPENDIX}

\section{Comparison with 2007-09 AEW Forecasts}

This work also assessed AEW intensity SD for the period of JAS 2007-09, the other period included in the Elless and Torn (2018) dataset. Given the 2007-09 and 2011-13 periods are associated with different model

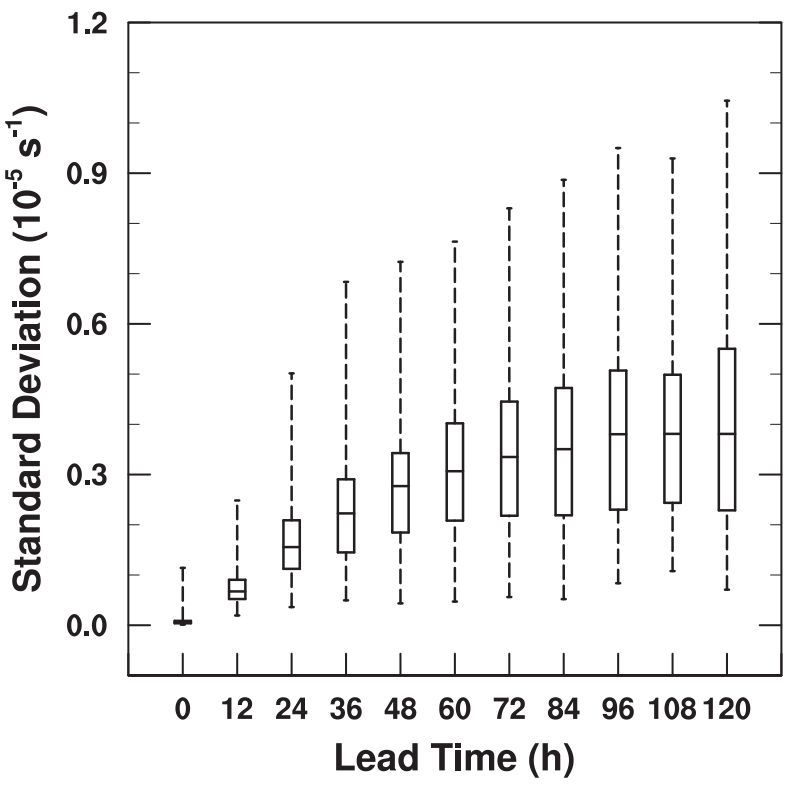

FIG. A1. As in Fig. 1, but for the 2007-09 period.

configurations and AEW evolution, it is imperative that they are assessed separately. The 2007-09 AEW intensity forecasts are characterized by near-zero SD at $0 \mathrm{~h}$, but quickly increases to a mean of $0.28 \times 10^{-5} \mathrm{~s}^{-1}$ (Fig. A1), which differs from the AEW intensity SD evolution during the first $48 \mathrm{~h}$ in the 2011-13 forecast period (cf. Fig. 1). These differences are likely related to the ECMWF's implementation of the ensemble data assimilation technique (e.g., Buizza et al. 2008) during 2010 model upgrades. This method was designed to generate additional initial condition uncertainty (particularly in the tropics), which explains the larger SD for early lead times during the 2011-13 period. Beyond $48 \mathrm{~h}$, the 200709 AEW intensity SD evolves in a similar manner to the 2011-13 period, suggesting that similar processes are controlling the forecast AEW intensity SD beyond 48 hours. The high and low SD forecasts during the 2007-09 period also highlighted differences in 0-h moisture and subsequent precipitation as the most significant differences between the two periods (not shown), consistent with the 2011-13 results. This suggests the source of AEW intensity SD differences at longer lead times are not impacted by previous model changes.

\section{REFERENCES}

Berry, G. J., and C. D. Thorncroft, 2005: Case study of an intense African easterly wave. Mon. Wea. Rev., 133, 752-766, https:// doi.org/10.1175/MWR2884.1.

— , and - 2012: African easterly wave dynamics in a mesoscale numerical model: The upscale role of convection. J. Atmos. Sci., 69, 1267-1283, https://doi.org/10.1175/JAS-D-11-099.1. 
Bougeault, P., and Coauthors, 2010: The THORPEX Interactive Grand Global Ensemble. Bull. Amer. Meteor. Soc., 91, 10591072, https://doi.org/10.1175/2010BAMS2853.1.

Brammer, A., and C. D. Thorncroft, 2015: Variability and evolution of African easterly wave structures and their relationship with tropical cyclogenesis over the eastern Atlantic. Mon. Wea. Rev., 143, 4975-4995, https://doi.org/10.1175/MWR-D15-0106.1.

Brown, D. P., 2013: Tropical cyclone report Hurricane Nadine (10 September-3 October 2012). NOAA/National Hurricane Center Tech. Rep. AL142012, 19 pp.

Buizza, R., M. Leutbecher, and L. Isaksen, 2008: Potential use of an ensemble of analyses in the ECMWF Ensemble Prediction System. Quart. J. Roy. Meteor. Soc., 134, 2051-2066, https:// doi.org/10.1002/qj.346.

Durran, D. R., and M. Gingrich, 2014: Atmospheric predictability: Why butterflies are not of practical importance. J. Atmos. Sci., 71, 2476-2488, https://doi.org/10.1175/JAS-D-14-0007.1.

_ and J. A. Weyn, 2016: Thunderstorms do not get butterflies. Bull. Amer. Meteor. Soc., 97, 237-243, https://doi.org/10.1175/ BAMS-D-15-00070.1.

Elless, T. J., and R. D. Torn, 2018: African easterly wave forecast verification and its relation to convective errors within the ECMWF ensemble prediction system. Wea. Forecasting, 33, 461-477, https://doi.org/10.1175/WAF-D-17-0130.1.

Halperin, D. J., and R. D. Torn, 2018: Diagnosing conditions associated with large intensity forecast errors in the Hurricane Weather Research and Forecasting (HWRF) Model. Wea Forecasting, 33, 239-266, https://doi.org/10.1175/WAF-D-170077.1.

Hsieh, J.-S., and K. H. Cook, 2007: A study of the energetics of African easterly waves using a regional climate model. J. Atmos. Sci., 64, 421-440, https://doi.org/10.1175/JAS3851.1.

Jackson, B., S. E. Nicholson, and D. Klotter, 2009: Mesoscale convective systems over western equatorial Africa and their relationship to large-scale circulation. Mon. Wea. Rev., 137, 1272-1294, https://doi.org/10.1175/2008MWR2525.1.

Janicot, S., and B. Sultan, 2001: Intra-seasonal modulation of convection in the West African monsoon. Geophys. Res. Lett., 28, 523-526, https://doi.org/10.1029/2000GL012424.

Janiga, M. A., and C. D. Thorncroft, 2013: Regional differences in the kinematic and thermodynamic structure of African easterly waves. Quart. J. Roy. Meteor. Soc., 139, 1598-1614, https:// doi.org/10.1002/qj.2047.

Komaromi, W. A., and S. J. Majumdar, 2014: Ensemble-based error and predictability metrics associated with tropical cyclogenesis. Part I: Basinwide perspective. Mon. Wea. Rev., 142, 2879-2898, https://doi.org/10.1175/MWR-D-13-00370.1.

_ , and —, 2015: Ensemble-based error and predictability metrics associated with tropical cyclogenesis. Part II: Waverelative framework. Mon. Wea. Rev., 143, 1665-1686, https:// doi.org/10.1175/MWR-D-14-00286.1.

Liebmann, B., and C. A. Smith, 1996: Description of a complete (interpolated) outgoing longwave radiation dataset. Bull. Amer. Meteor. Soc., 77, 1275-1277, https://doi.org/10.1175/ 1520-0477-77.6.1274.

Lorenz, E. N., 1969: The predictability of a flow which possesses many scales of motion. Tellus, 21, 289-307, https://doi.org/ 10.3402/tellusa.v21i3.10086.

McMurdie, L. A., and B. Ancell, 2014: Predictability characteristics of landfalling cyclones along the North American west coast. Mon. Wea. Rev., 142, 301-319, https://doi.org/10.1175/MWRD-13-00141.1.
Norquist, D. C., E. E. Recker, and R. J. Reed, 1977: The energretics of African wave disturbances as observed during Phase III of GATE. Mon. Wea. Rev., 105, 334-342, https://doi.org/10.1175/ 1520-0493(1977)105<0334:TEOAWD>2.0.CO;2.

Payne, S. W., and M. M. McGarry, 1977: The relationship of satellite inferred convective activity to easterly waves over West Africa and the adjacent ocean during phase III of GATE. Mon. Wea. Rev., 105, 413-420, https://doi.org/10.1175/15200493(1977)105<0413:TROSIC > 2.0.CO;2.

Reed, R. J., D. C. Norquist, and E. E. Recker, 1977: The structure and properties of African wave disturbances as observed during phase III of GATE. Mon. Wea. Rev., 105, 317-333, https://doi.org/10.1175/1520-0493(1977)105<0317: TSAPOA $>2.0 . \mathrm{CO} ; 2$.

Rios-Berrios, R., R. D. Torn, and C. A. Davis, 2016a: An ensemble approach to investigate tropical cyclone intensification in sheared environments. Part I: Katia (2011). J. Atmos. Sci., 73, 71-93, https://doi.org/10.1175/JAS-D-15-0052.1.

$\longrightarrow, \ldots$, and ——, 2016b: An ensemble approach to investigate tropical cyclone intensification in sheared environments. Part II: Ophelia (2011). J. Atmos. Sci., 73, 1555-1575, https:// doi.org/10.1175/JAS-D-15-0245.1.

Straub, K. H., and G. N. Kiladis, 2003: The observed structure of convectively coupled Kelvin waves: Comparison with simple models of coupled wave instability. J. Atmos. Sci., 60, 1655-1668, https://doi.org/10.1175/1520-0469(2003)060<1655: TOSOCC $>2.0 . \mathrm{CO} ; 2$.

Sultan, B., S. Janicot, and A. Diedhiou, 2003: The West African monsoon dynamics. Part I: Documentation of intraseasonal variability. J. Climate, 16, 3389-3406, https://doi.org/10.1175/ 1520-0442(2003)016<3389:TWAMDP $>2.0 . \mathrm{CO} ; 2$.

Thorncroft, C. D., and B. J. Hoskins, 1994: An idealized study of African easterly waves. I: A linear view. Quart. J. Roy. Meteor. Soc., 120, 953-982, https://doi.org/10.1002/qj.49712051809.

, and D. P. Rowell, 1998: Interannual variability of African wave activity in a general circulation model. Int. J. Climatol., 18, 1305-1323, https://doi.org/10.1002/(SICI)1097-0088(1998100) 18:12<1305::AID-JOC281>3.0.CO;2-N

Tomassini, L., D. J. Parker, A. Stirling, C. Bain, C. Senior, and S. Milton, 2017: The interaction between moist diabatic processes and the atmospheric circulation in African Easterly Wave propogation. Quart. J. Roy. Meteor. Soc., 143, 3207-3227, https:// doi.org/10.1002/qj.3173.

Torn, R. D., 2010: Ensemble-based sensitivity analysis applied to African easterly waves. Wea. Forecasting, 25, 61-78, https:// doi.org/10.1175/2009WAF2222255.1.

— J. S. Whitaker, P. Pegion, T. M. Hamill, and G. J. Hakim, 2015: Diagnosis of the source of GFS medium-range track errors in Hurricane Sandy (2012). Mon. Wea. Rev., 143, 132-152, https://doi.org/10.1175/MWR-D-14-00086.1.

van der Linden, R., A. H. Fink, J. G. Pinto, T. Phan-Van, and G. N. Kiladis, 2016: Modulation of daily rainfall in southern Vietnam by the Madden-Julian oscillation and convectively coupled equatorial waves. J. Climate, 29, 5801-5820, https://doi.org/ 10.1175/JCLI-D-15-0911.1.

Ventrice, M. J., and C. D. Thorncroft, 2013: The role of convectively coupled atmospheric kelvin waves on African easterly wave activity. Mon. Wea. Rev., 141, 1910-1924, https:// doi.org/10.1175/MWR-D-12-00147.1.

,- , and P. E. Roundy, 2011: The Madden-Julian oscillation's influence on African easterly waves and downstream tropical cyclogenesis. Mon. Wea. Rev., 139, 2704-2722, https:// doi.org/10.1175/MWR-D-10-05028.1. 
Wheeler, M., and G. N. Kiladis, 1999: Convectively coupled equatorial waves: Analysis of clouds and temperature in the waveumber-frequency domain. J. Atmos. Sci., 56, 374-399, https://doi.org/10.1175/1520-0469(1999)056<0374:CCEWAO> 2.0.CO;2.

Yasunaga, K., and B. Mapes, 2012: Differences between more divergent and more rotational types of convectively coupled equatorial waves. Part II: Composite analysis based on spact-time filtering. J. Atmos. Sci., 69, 17-34, https://doi.org/ 10.1175/JAS-D-11-034.1.

Zhang, C., 2005: Madden-Julian oscillation. Rev. Geophys., 43, RG2003, https://doi.org/10.1029/2004RG000158.

Zhang, F., C. Snyder, and R. Rotunno, 2003: Effects of moist convection on mesoscale predictability. J. Atmos. Sci., 60 , 1173-1185, https://doi.org/10.1175/1520-0469(2003)060<1173: EOMCOM $>2.0 . \mathrm{CO} ; 2$. 